\title{
Equilibration of centrifugally unstable vortices: a review
}

\author{
G. F. Carnevale ${ }^{1}$, R. C. Kloosterziel ${ }^{2,3}$ and P. Orlandi ${ }^{4}$ \\ ${ }^{1}$ Scripps Institution of Oceanography, University of California, San Diego, La Jolla, CA 92093, USA \\ ${ }^{2}$ School of Ocean and Earth Science and Technology, University of Hawaii, Honolulu, HI 96822, USA \\ ${ }^{3}$ Royal Netherlands Institute for Sea Research, PO Box 59, 1790 AB Texel, the Netherlands \\ ${ }^{4}$ Dipartimento di Meccanica e Aeronautica, University of Rome, "La Sapienza," \\ via Eudossiana 18, 00184 Roma, Italy
}

\begin{abstract}
In three-dimensional flow, a vortex can become turbulent and be destroyed through a variety of instabilities. In rotating flow, however, the result of the breakup of a vortex is usually a state comprising several vortices with their axes aligned along the ambient rotation direction. This article is a review of our recent work on how the combined effect of centrifugal and barotropic instabilities can breakup a vortex and lead to its reformation in a predictable way even though an intermediate stage in the evolution is turbulent. Centrifugal instability tends to force the unstable vortex into a turbulent state that mixes absolute angular momentum in such a way as to precondition the flow for a subsequent barotropic instability. A method for predicting the redistribution of angular momentum and the resulting velocity profile is discussed. The barotropic instability horizontally redistributes the component of vorticity that is aligned along the ambient rotation vector, resulting in the final byproducts of the instability, which are stabilized by the effects of ambient rotation. A prediction scheme that puts the tendencies of these two instabilities together proves to be very reliable.
\end{abstract}

Keywords: vortex dynamics, stability, rotating flow

\section{Introduction}

Vortices and currents in the oceans are subject to a variety of important instabilities including centrifugal, barotropic and baroclinic instabilities. One of our goals has been to achieve an understanding of how these instabilities participate in the formation and maintenance of observed vortices and currents. In experiments, both laboratory and numerical, which can be designed such that only centrifugal and barotropic instability are relevant, a great deal of insight has been achieved, and it is now possible to predict, to a great extent, the results of the combined effects of centrifugal and barotropic instabilities. The purpose of this article is to review this development in the case of anticyclonic vortices in some detail. The cases of unstable cyclonic vortices and planar currents have also been dealt with rather successfully, and we shall point the reader to the appropriate literature for those results.

Our motivation for a detailed study of the evolution of centrifugal instability in the case of anticyclonic vortices came from the laboratory studies discussed in Kloosterziel and van Heijst (1991)[1]. The basic experiment is illustrated schematically in Fig. 1. The experiments were performed in a tank rotating about a vertical axis with angular velocity $\Omega$ and containing a layer of water. A hollow cylinder, of height more than the depth of the water, was inserted into the tank and left standing vertically. A vortex was then created by stirring within the cylinder either cyclonically (in the same direction of the rotation of the tank) or anticyclonically. The cylinder was lifted vertically, leaving behind a vortex in the quiescent surrounding fluid. In the case of cyclonic vortices, the subsequent evolution was relatively smooth, more or less two-dimensional, that is independent of depth. As shown in figure 2, in this case the vortex 


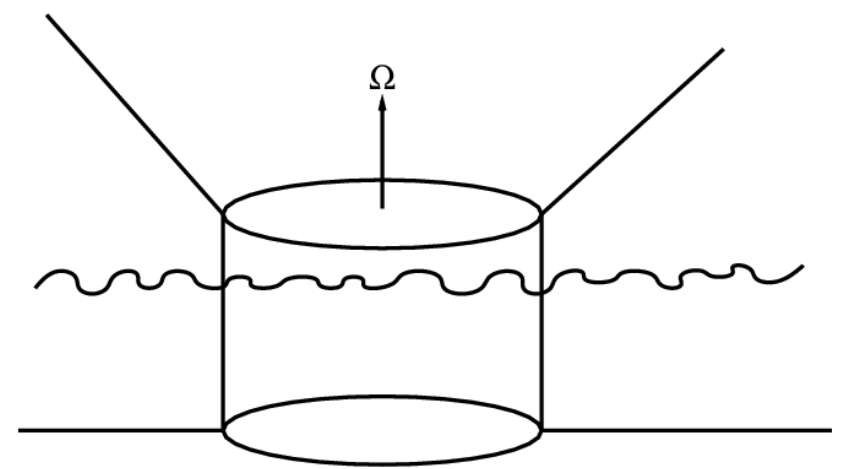

(a)

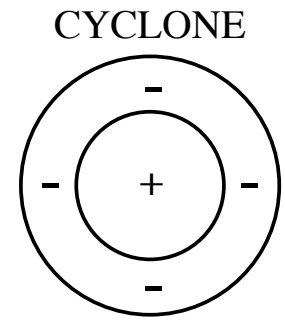

$\omega_{z}$

ANTICYCLONE

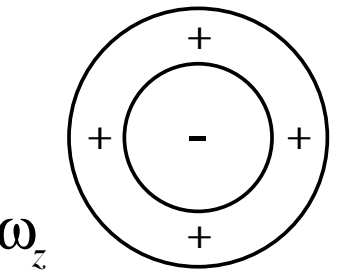

(b)

Figure 1. Schematics illustrating the basic laboratory experiments investigated in Kloosterziel and van Heijst (1991)[1]. (a) A hollow cylinder is placed vertically within a tank rotating about a vertical axis. The tank is filled with water to a level which is lower than the height of the inserted hollow cylinder. The flow is stirred either cyclonically or anticyclonically within the hollow cylinder and allowed to adjust to a smooth flow, at which point the hollow cylinder is lifted vertically by the agency of the supports attached to its upper rim. (b) The vertical vorticity field within the hollow cylinder just before it is removed from the tank is either that of a cyclone or an anticyclone. Since these are isolated vortices with no flow initially outside the cylinder, they take on the form of a core of cyclonic/anticyclonic vorticity surrounded by an annulus of vorticity of the opposite sign. The total vorticity within the cylinder must vanish since the velocity on the cylinder is zero.

typically evolved into a cyclonic vortex with two satellite anticyclonic vortices, one on either side of the core cyclone. This configuration is referred to as a tripole (see e.g. van Heijst and Kloosterziel (1989)[2], van Heijst et al.(1991)[3]). In the cases in which the initial vortex within the cylinder was an anticyclone, the release was followed by a brief period of vigorous turbulent motion throughout the vortex column followed by the vortex being torn apart resulting in two dipolar vortices that moved away from each other as they became more and more two-dimensional Kloosterrziel and van Heijst (1991)[1]. An example of this type of evolution is shown in figure 3.

If the flow in these experiments were at all times two-dimensional, then except for the sign of the velocity field, one would expect that the behavior would be the same for cyclones and anticyclones, for then there is nothing in the physics that would break the (anti-)symmetry of the flows. This led Kloosterziel and van Heijst (1991) [1] to conclude that the early three-dimensional turbulent motion so prominent in the anticyclonic case leads to the difference between the evolution of cyclones and anticyclones. At the moderate initial velocities in these experiments, one would expect anticyclones but not cyclones to be vulnerable to centrifugal instability. Thus, Kloosterziel and van Heijst argued that it was centrifugal instability that somehow changed the vorticity distribution in such a way as to make the results very different in the two cases.

The redistribution of vorticity from a monopolar form as shown in figure $1 \mathrm{~b}$ into forms such as dipoles or tripoles as seen in the laboratory experiments can be accomplished by barotropic instability. Such an instability can produce a variety of complex forms, most of which are unstable and typically degenerate into monopoles, dipoles and tripoles. We investigated this in detail in Carnevale and Kloosterziel (1994)[4]. Indeed, one of these intermediate complex forms, a quadrapole, was already observed in the laboratory by Kloosterziel and van Heijst (1991)[1]. Additionally, theoretically it should have been possible even for an anticyclone to become a tripole. In fact, we found an example of this in later laboratory experiments (Carnevale et al. (2011)[5]) as shown here in figure 4, but this was a rare event.

Through three-dimensional numerical simulations, Orlandi and Carnevale (1999)[6] investigated the dual role played by barotropic and centrifugal instabilities. At high Reynolds numbers, simulations with no ambient rotation show that a perturbed vortex column will just degenerate into 3D turbulence (see for example Carnevale et al.1997 [7]). With ambient rotation, however, the flow will eventually tend to columnar (i.e. vertically uniform) flow, in accord with the Taylor-Proudman theorem. Thus it is not a surprise that after the initial rather turbulent phase of the anticyclonic vortex, the flow becomes two-dimensional (columnar). Orlandi and Carnevale (1999)[6] confirmed Kloosterziel and van Heijst's (1991)[1] hypothesis that the centrifugal instability set up the conditions for the barotropic instability to create a pair of dipoles in the anticyclonic case rather than a compact tripole as in the cyclonic case. Centrifugal instability was shown to increase the horizontal velocity gradients in the flow and this triggered the barotropic instability that led to double dipoles. The precise nature and effect of the centrifugal instability was shown to depend on the 
details of the initial distribution of vorticity. The possible evolution of an anticyclone into a tripole as well as double dipoles after a centrifugal instability was also confirmed. Additionally, the intermediate form a quadrapole was also produced.

Thus we had shown that 3D numerical simulation could capture all of the phenomena that had been observed in in the laboratory. This was not entirely satisfying because it was not yet possible to predict, from a knowledge of only the initial state, what structures, intermediate and final, would emerge from an initially centrifugally unstable vortex. We proceeded to make a thorough study of centrifugal instability. In Kloosterziel et al.(2007)[8], we found that in the limit of infinite Reynolds number, the distribution of vorticity could be predicted precisely knowing only the initial distribution. This was the key to predicting the combined effect of both centrifugal and barotropic instabilities. We were then able to devise a new scheme that allowed us to predict the evolution of the full 3D flow based solely on this new knowledge of the precise effect of centrifugal instability and the two-dimensional evolution of barotropic instability.

In what follows, we shall explain how this prediction scheme works and show evidence for its effectiveness. In section 2 , we review the necessary background information about the barotropic instability. In section 3 , we discuss the method of predicting the precise result of centrifugal instability in the limit of infinite Reynolds number. In section 4, we show how this new knowledge of how centrifugal instability equilibrates can be used in the prediction of the outcome of the combined centrifugal and barotropic instabilities. In section 5, we conclude with a discussion of how the methods discussed here have been extended to initially planar barotropic currents. 

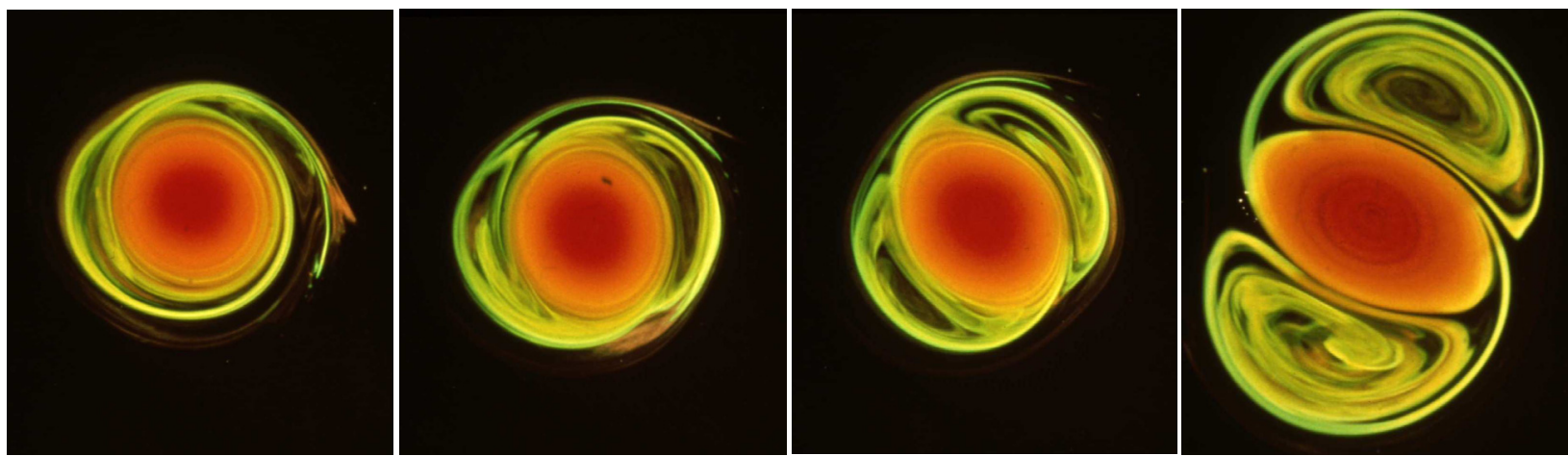

Figure 2. Formation of a tripolar vortex with a cyclonic core. The camera looks down along the axis of rotation of the tank and is fixed in the rotating frame of reference. The color was added to the initial monopolar vortex in such a way that the core was orange and the outer edges were yellow. This resulted in the core cyclone being orange and the anticyclonic satellites yellow. (Reproduced from van Heijst et al.(1991)[3] with permission from Cambridge University Press)
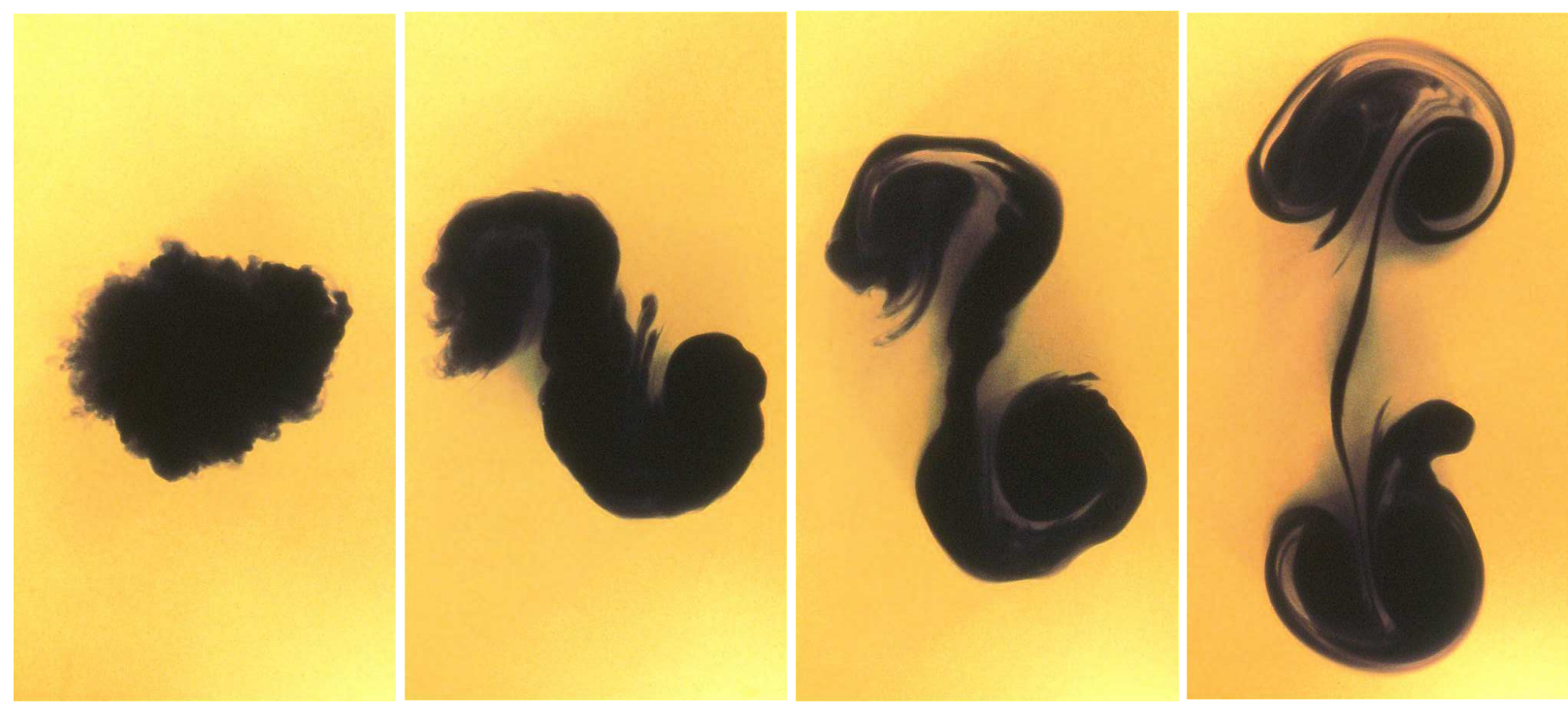

Figure 3. Transformation of an anti-cyclone into a pair of dipoles in a rotating tank experiment. After a turbulent phase, the flow becomes increasingly two-dimensional, that is invariant in the vertical direction. In the end state there are two dipolar vortices propagating away from each other. These images have previously appeared on the front cover of the Journal of Fluid Mechanics, vol. 669 (2011) (Reproduced with the permission of Cambridge University Press).
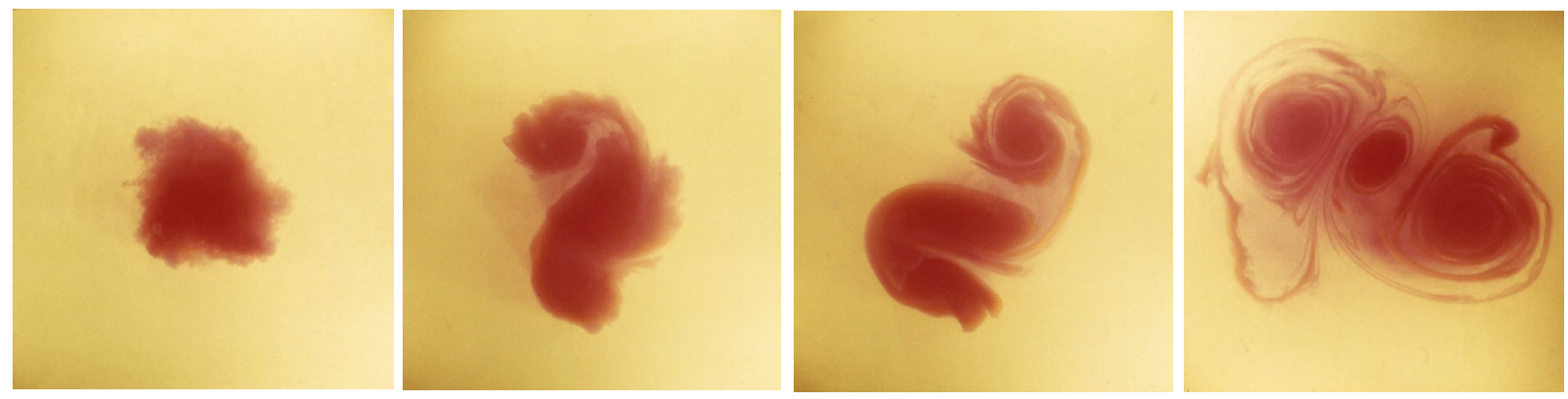

Figure 4. Transformation of an anti-cyclone into a tripole in a rotating tank experiment. After a turbulent phase, the flow becomes increasingly two-dimensional, that is invariant in the vertical direction. In the end state there is a tripole with an anticyclonic core and two cyclic satellites. 


\section{Barotropic instability}

The role of the barotropic instability in the evolution of the flows shown in Figures 2-4 is the horizontal redistribution of vertical vorticity from the early circular form into the final tripoles or double-dipoles. This instability is essentially two-dimensional and results when horizontal velocity gradients are sufficiently steep. Although Rayleigh (1880)[9] and others (see Drazin and Reid, 1981 [10]) have given necessary conditions for the instability, there are no comparable simple sufficient conditions. Analytical progress toward understanding the barotropic instability has been made using normal modes analysis on simple vorticity step profiles (e.g. Stern, 1987[11]; Flierl, 1988[12]; Kloosterziel and Carnevale, 1992[13]). For more general vorticity profiles, we use numerical methods to obtain the normal modes and their growth rates.

Various continuous profiles have been used to model the initial flow inside the hollow cylinder of the laboratory experiments. One particularly well studied profile (see Carton et al.1989[14]; Carton and Flierl (1989)[15]; Orlandi and van Heijst, 1992[16]; Carton and Legras, 1994[17]; Carnevale and Kloosterziel, 1994[4]; Orlandi and Carnevale, 1999[6]; Kloosterziel and Carnevale, 1999[18]; Gallaire and Chomaz, 2003[19]) is given by

$$
V(r)=V_{0} \frac{r}{2 \ell} \exp \left(-(r / \ell)^{\alpha}\right),
$$

where the velocity $V$ is the azimuthal velocity in a polar coordinate system in the horizontal plane, a cross section of the cylindrical coordinate system that we will use for the full 3D problem. $r$ is the distance from the center of the vortex in that plane. Since we are concerned in this section only with the barotropic flow, no vertical coordinate is introduced at this point. Non-dimensionalizing with the vortex length-scale $\ell$ and the advective time-scale $\ell /\left|V_{0}\right|$, we can write the velocity profile as

$$
V(r)= \pm \frac{r}{2} \exp \left(-r^{\alpha}\right)
$$

where the $+/-$ sign holds for the cyclone/anticyclone. Since there is no vertical variation involved in this instability, the problem can be treated entirely in the framework of 2D fluid dynamics, and the rotation rate of the environment does not appear in the evolution equation. Thus, although we call positive/negative vorticity cyclonic/anticyclonic, this a mere convenience for when we will return to the full 3D problem.

The vertical vorticity associated with this horizontal vorticity distribution is given by

$$
\omega_{z}(r)= \pm\left(1-\frac{\alpha}{2} r^{\alpha}\right) \exp \left(-r^{\alpha}\right) .
$$

and has vorticity \pm 1 at the center of the vortex, i.e. at $r=0$. The positive parameter $\alpha$ controls the gradient of velocity outside the core of the vortex. This is illustrated in Fig. 5 where profiles for $V$ with values of $\alpha$ running from 3 to 7 are shown. As $\alpha$ increases, the gradient of velocity at the boundary of the core of the vortex increases, and, correspondingly, so does the magnitude of the change in vorticity between the center of the vortex and the outer annulus of oppositely signed vorticity.

The barotropic instability of a vorticity distribution with profile (3) has been the subject of many studies (e.g. Carton and McWilliams (1989)[15], Orlandi and van Heijst (1992)[16], Carnevale and Kloosterziel (1994)[4] and Kloosterziel and Carnevale (1999)[18]). Consider perturbing the circularly symmetric vorticity distribution (3) with a small perturbation of the form

$$
\omega_{z}{ }^{\prime}=A \cos (\mathrm{m} \theta)+B \sin (\mathrm{m} \theta),
$$

where $\theta$ is the angle in our cylindrical coordinate system, $A$ and $B$ are constants, and $m$ is the azimuthal wavenumber of the perturbation. Depending on the value of the steepness parameter $\alpha$, the perturbation will either not grow at all or grow exponentially. Kloosterziel and Carnevale (1999) reported the growth rates for perturbations with wavenumbers $\mathrm{m}=2,3$ and 4 for a range of $\alpha$. Those results are reproduced in Fig. 6 . In that figure, we see that for $\alpha \lesssim 1.85$ there is no barotropic instability. For $1.85 \lesssim \alpha \lesssim 3$ only mode 2 can grow. For $3 \lesssim \alpha \lesssim 4$ only modes 2 and 3 can grow. For $4 \lesssim \alpha$ modes 2,3 and 4 can all grow, and presumably so can higher wavenumber modes depending on the size of $\alpha$, with more modes becoming unstable as $\alpha$ is increased.

In simulations of the evolution of the vortex with a perturbation of a single azimuthal mode, nonlinear effects eventually become important and the vortex is transformed into a structure with a central core of the same sign of 

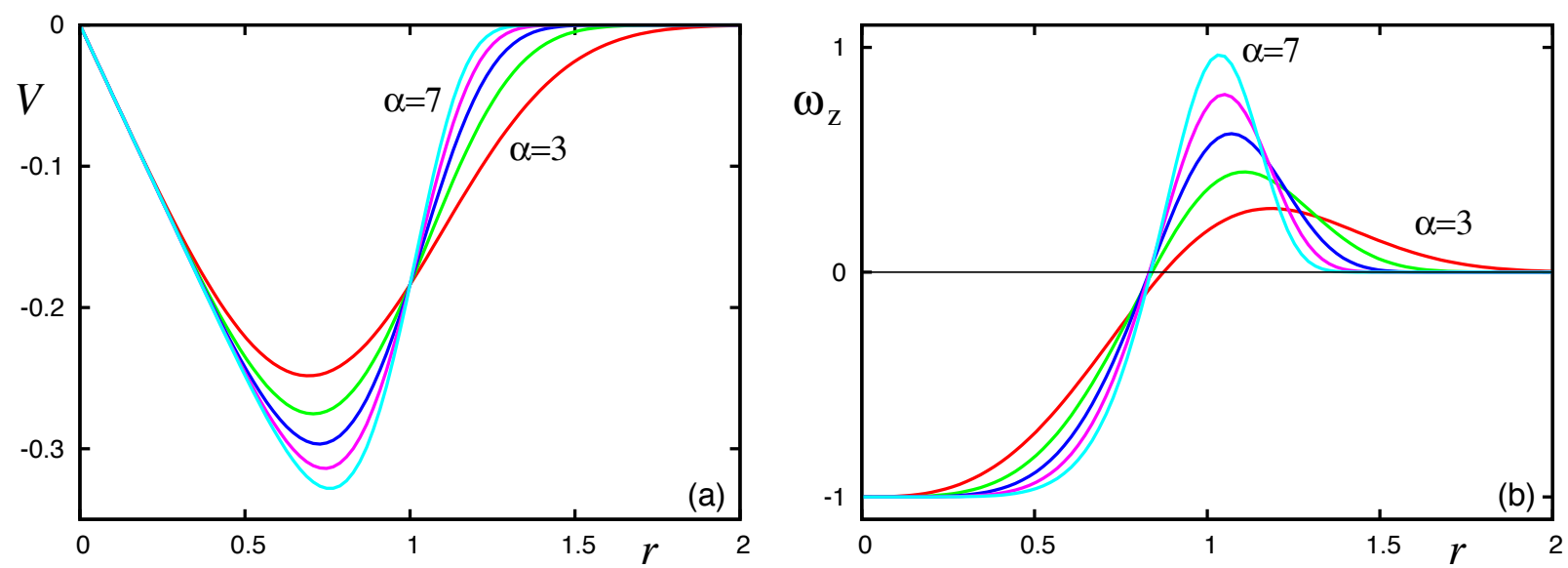

Figure 5. Nondimensional model profiles for anticyclones for values of $\alpha$ in unit increments from 3 to 7: (a) velocity from formula (1); (b) vertical vorticity from formula (3)

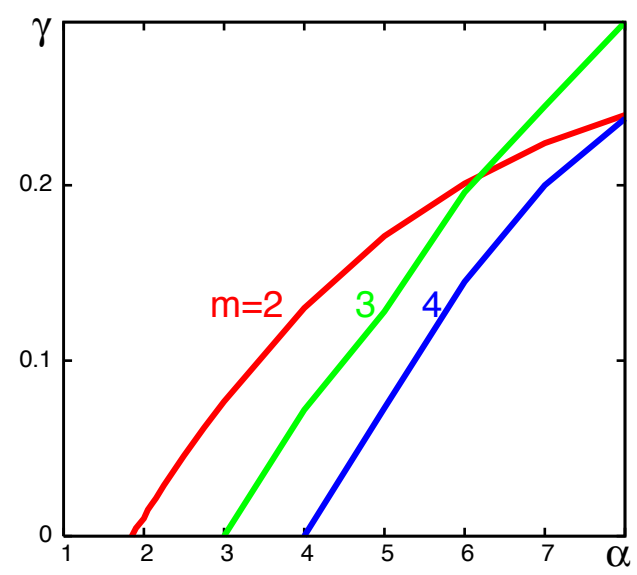

Figure 6. Non-dimensional exponential growth rate $\gamma$ of barotropic instability for azimuthal modes $\mathrm{m}=2,3,4$ as in Carnevale and Kloosterziel (1994)[4].

vorticity as in the core of the original unperturbed vortex. The symmetry of the structure is that of the given azimuthal perturbation. The core takes on a geometrical shape of that symmetry and it is surrounded by m cyclones of vorticity of the opposite sign (see Fig. 7). These structures are not all stable. Carnevale and Kloosterziel (1994)[4] found the tripole $(\mathrm{m}=2)$ and quadrupole $(\mathrm{m}=3)$ to be stable, but all of the higher order 'multipoles' were unstable. In the instability of the 'multipole, fluctuations bring two of the outer satellite vortices into closer proximity resulting in their merger due to the tendency of like signed vortices to merge (see Melander and McWilliams, 1987[20]; Melander et al.1988[21]; Carnevale et al.1991a[22] and Carnvale et al.1991b[23]). After such a merger, the structure typically breaks up into a set of dipoles and monopoles.

For $1.85 \lesssim \alpha \lesssim 3$, with a small perturbation made up of many different azimuthal modes, the result is typically a stable tripole since only mode 2 is unstable. However, for $3 \lesssim \alpha \lesssim 4$ and an initial perturbation including modes 3 and 4, modes 2, 3 and 4 will all grow and typically the tripole will break up into two dipoles. For higher values of $\alpha$, the evolution can be very complex, even early on, because many different modes will be unstable with not very disparate growth rates.

As discussed in the introduction, cyclonic and anticyclonic initial vortices with similar distributions of vorticity (apart from the sign) give rather different results in the rotating tank, with the cyclones producing tripoles and the anticyclones typically resulting in double-dipoles. Given that in barotropic (i.e. 2D) flow, there is nothing to break the 


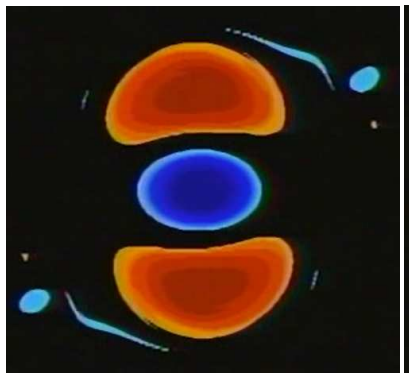

(a)

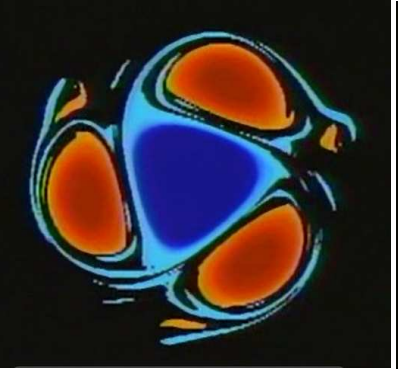

(b)

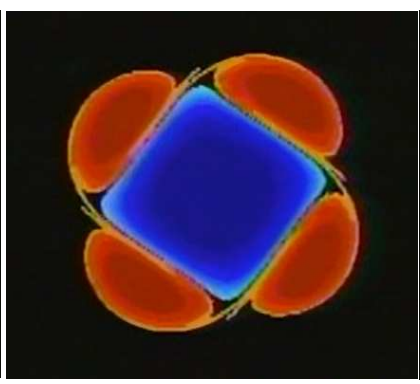

(c)

$\mathrm{m}=4$

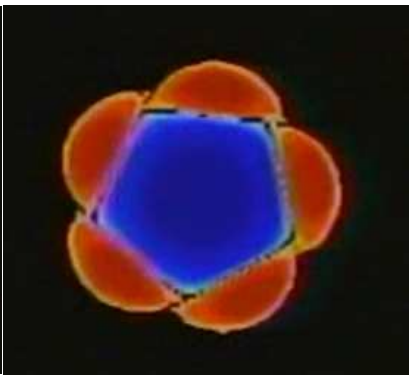

(d)

$\mathrm{m}=5$

Figure 7. Shaded contour plots of vertical vorticity $\omega_{z}$ from simulations of barotropic instability starting from a circularly symmetric vortex plus a perturbation $\omega_{z}{ }^{\prime}=A \cos (\mathrm{m} \theta)+B \sin (\mathrm{m} \theta)$ with: (a) $\mathrm{m}=2$, (b) $\mathrm{m}=3$, (c) $\mathrm{m}=4$ and (d) $\mathrm{m}=5$. This $2 \mathrm{D}$ spectral simulation is discussed in detail in Carnevale and Kloosterziel (1994)[4].

symmetry between the evolution of cyclones and anticyclones, we must next discuss what is it about the 3D flow that makes this distinction. From the discussion in this section, the key seems to be in understanding how the anticyclonic profiles may be steepened (analogous to producing a higher $\alpha$ ) by centrifugal instability.

\section{Centrifugal instability}

The essence of centrifugal instability can be understood in the context of purely circularly symmetric flows. A criterion for linear instability of a circularly-symmetric barotropic vortex can be obtained by considering energy changes due to perturbations of such a vortex. An important constraint in such flows is that the absolute angular momentum $L$ is conserved on particles. $L$ can be written as $L=r(v+\Omega r)$, where $v$ is the azimuthal velocity and $\Omega$ is the angular rotation rate of the environment. Centrifugal instability occurs if and only if $L^{2}$ decreases somewhere in the flow, that is

$$
\text { Instability } \Leftrightarrow \frac{d L^{2}}{d r}=2 L L_{r}<0,
$$

(see Rayleigh, 1916[24]; Drazin and Reid, 1981[10]; Kloosterziel and van Heijst, 1991[1]).

Unlike the case of barotropic flow, when the flow is $3 \mathrm{D}$, even if constrained to be azimuthally symmetric, the rotation rate of the environment becomes an important variable. It becomes convenient then to define the Rossby number $R o$ of the flows that we will be considering. Using the parameters of the basic profile defined by (1), we define

$$
R o=V_{0} / f \ell \text { with } f=2 \Omega \text { the Coriolis parameter. }
$$

Note that the Rossby number is signed with positive/negative Ro corresponding to cyclones/anticyclones. Then, applying criterion (5) to the basic vortex (1), we find that this vortex is centrifugally unstable for sufficiently strong anticyclones with

$$
\text { Ro }<-1
$$

or for sufficiently strong cyclones with

$$
R o>R o_{\mathrm{cr}}
$$

where

$$
R o_{\mathrm{cr}}=\frac{2}{\alpha} \exp \left(\frac{\alpha+2}{\alpha}\right)>0 .
$$

(see Carnevale and Orlandi (1999)[6]).

\subsection{Nonlinear equilibration: Anticyclones}

Fig. 8 shows a plot of $L$ for an example of our profile with $R o=-4$ and $\alpha=3$, values for which the vortex is unstable. There is a range of $r$ for which $L^{2}$ is decreasing: $\left(r_{-}<r<r_{+}\right)$. In this region, the flow is linearly unstable. From this diagram, we see that $r_{-}$is the point where $L$ achieves its minimum. We do not have a closed form 


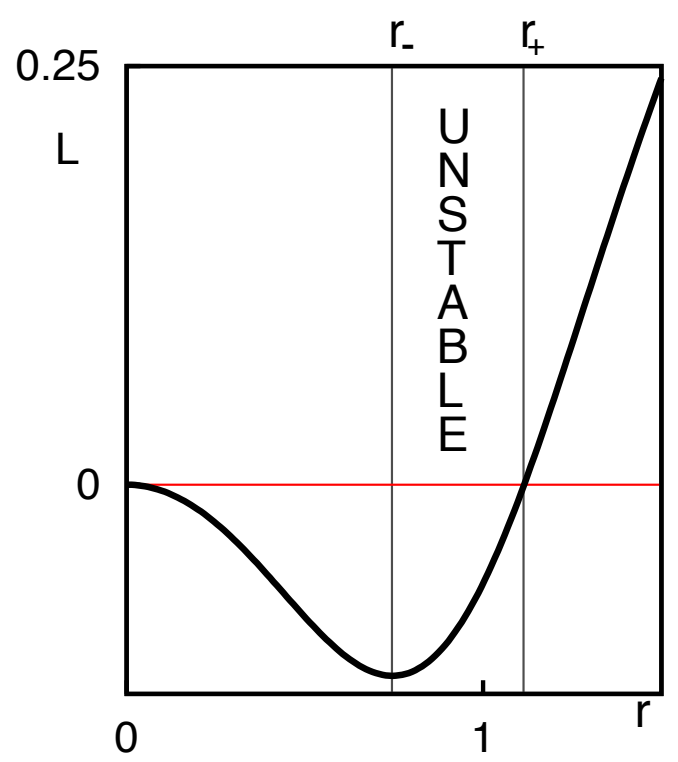

Figure 8. A graph of the absolute angular momentum $L_{0}=r(V+\Omega r)$ in the case of an anticyclone with profile (1) with Ro $=-4$ and $\alpha=3$. The limits of the linear instability range, $r_{-}$and $r_{+}$, are marked by the thin vertical lines.

expression for this point, but its value is easily calculated numerically. $r_{+}$is the point where $L=0$, and this is given by $r_{+}=(\ln |R o|)^{1 / \alpha}$.

A simulation of the evolution of this anticyclone is shown in Fig. 9. The initial condition for the simulation is the vortex given by (1) (with the vertical dimension added) with a small-amplitude small-scale (grid-scale) random perturbation. The plots show the azimuthal vorticity in a horizontal cross section. This simulation and all those discussed below were performed with a finite-difference cylindrical-coordinate code that is described in detail in Orlandi (2000)[25]. It is an energy conserving code that can be run in azimuthally symmetric mode or fully 3D mode. In the figure, only a portion of the computational domain is shown, the region where the most intense activity occurs.

In panel (a) of Fig. 9, we see the effects of the linear growth of the most unstable modes of the instability. The linear dynamics produces a vertical stack of vortex rings of azimuthal vorticity, similar to the well-known TaylorCouette vortices (see e.g. Drazin and Reid 1981[10]). The sign of the vorticity alternates from one ring to the next in the stack. These vortices all form within the region of linear instability $r \in\left(r_{-}, r_{+}\right)$delimited by the vertical dotted lines in the figure. There are variations in the shape of the vortices and their positions in $r$ due to the finite and random nature of the initial perturbation. The vertical wavenumber of the most unstable mode is selected by a combination of the shape of the initial profile and the Reynolds number of the flow as discussed in detail by Kloosterziel, Carnevale and Orlandi (2007)[8] and Kloosterziel and Carnevale (2008)[26]. For the profile used here, we can precisely define the Reynolds number as

$$
R e=\frac{V_{0} \ell}{v},
$$

where $v$ is the viscosity of the flow. The most unstable mode for inviscid centrifugal instability has infinite vertical wavenumber, that is the thickness of the vortices in the stack shown in panel (a) of Fig. 9 would be infinitesimal. In Kloosterziel, Carnevale and Orlandi (2007)[8] we found through numerical simulations that for finite Re the most unstable vertical wavenumber (and hence the number of vortex rings in the stack) increases as $R e^{1 / 3}$. This was explained theoretically by S.D. Griffiths in the appendix in Kloosterziel and Carnevale (2008)[26]. If the dynamics were purely linear (i.e. if there were no advective interaction between the rings), then the amplitude of the rings would continue to increase exponentially without limit, and the stack would appear more and more regular as the fastest growing mode dominates over all others.

As the amplitude of the vortex rings continues to grow, nonlinear effects become more important and after a certain point control the evolution. In Fig. 9b, we see a stage in the evolution in which nonlinear interaction (through advec- 

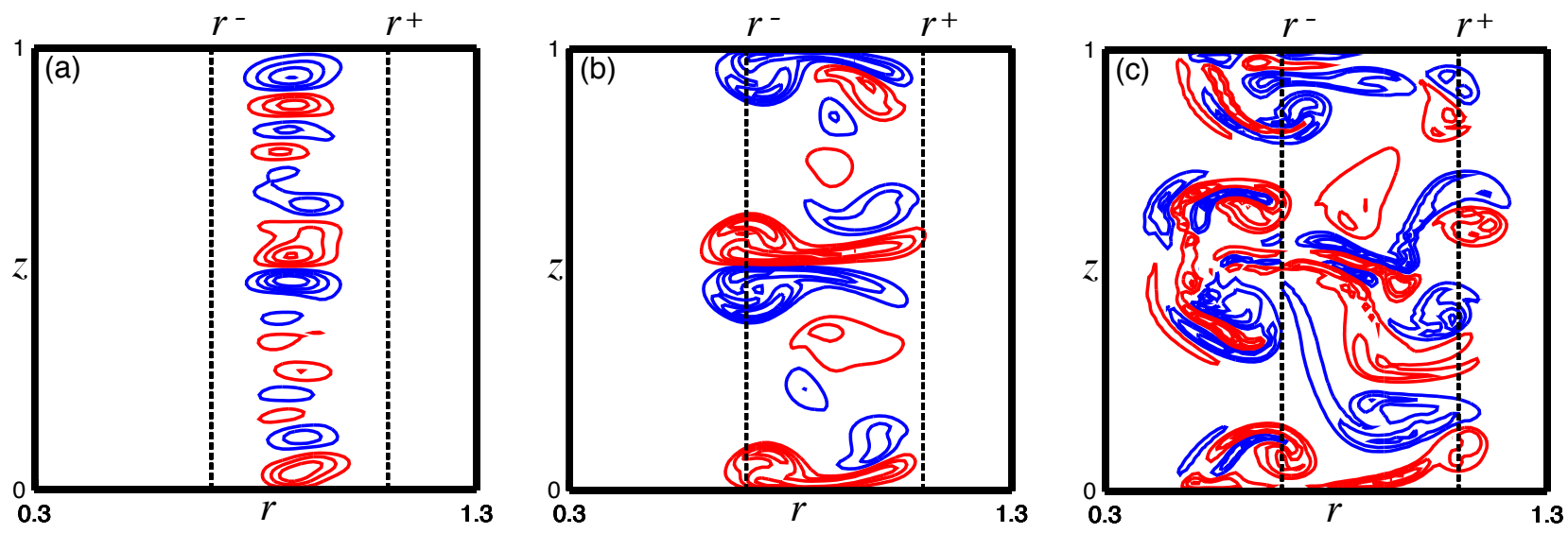

Figure 9. Contour plots of azimuthal vorticity $\omega_{\theta}$ showing the centrifugal instability of a barotropic anticyclone. The simulation allows no variation in the azimuthal direction. The images are vertical cross sections. Only a portion of the radial computational range of the simulation is shown. $r_{-}$ and $r_{+}$are the limits of the linear instability range which is bounded by the vertical dashed lines. The sequence of panels represent times (a) 28 , (b)56 and (c) 64. Red/blue (or grey/black in the greyscale version) contours represent positive/negative values of $\omega_{\theta}$ with contour interval 0.003 . (Reproduced from Carnevale et al.(2011)[5] with permission from Cambridge University Press)

tion) between the rings has created dipolar vortices. In that panel, one can see a very typical pattern for centrifugal instability in which a particular vortex, say one of positive azimuthal vorticity, couples with two vortices (one above and one below) simultaneously. Coupling with the one below (of negative vorticity), it forms a dipolar head 'pointing' towards the center of the initial basic vortex (i.e. toward $r=0$ ). Coupling with the one above (a different vortex of negative vorticity) it forms a dipolar tail 'pointing' away from the center of the basic vortex (i.e. toward $r=\infty$ ). By 'pointing' we mean to indicate the direction in which the dipolar structure is propagating. Dipoles propagate by the mutual advective interaction of the two oppositely signed vortices that comprise the two vortex structure. Since the vorticity indicated in Fig. 9 is azimuthal vorticity, a dipole with positive vorticity on top and negative vorticity on the bottom will propagate inwardly toward $r=0$ because the vortex on top produces clockwise motion around its center and 'pushes' the negative vortex below in the direction of decreasing $r$, while the negative vortex on the bottom produces counterclockwise flow about its center and hence moves the positive vortex above it also toward decreasing $r$. If the position of the vortices in the dipole are interchanged, the propagation would then be toward increasing $r$ by the same sort of argument. As the dipolar heads and dipolar tails propagate in opposite directions, they 'pull' out the vortices involved into thin filaments connecting the propagating dipolar structures.

The growth of perturbation vorticity and the nonlinear interaction between the vortices will continue until nonlinear effects sufficiently change the initial profile so that the instability ceases or saturates. This must somehow involve producing a profile in which there is no region with $L^{2}$ decreasing, that is in which there is no linearly unstable region. In Fig. 9c, we see that the propagation of the dipolar heads and tails and their interactions has resulted in a very complicated flow. Notice, as is already evident in panel (b), that the propagation of the dipoles allows the effects of the instability to be felt beyond the range of the initially unstable region. Since $L$ is transported on fluid particles, panel (c) indicates that the flow is actively mixing $L$ and, very importantly, we see that this mixing goes beyond the initial instability region. This raises the essential questions: how efficient is the mixing, how far does it extend and how does it change the profile of $L$ so that the instability is killed?

We can make progress toward answering these questions by looking at vertically averaged profiles of $L$ at times when the amplitudes of the perturbation vortices have decayed to values much lower than their peak values and the vertically averaged velocity field has essentially stabilized. Such profiles are shown in Fig. 10a for various values of the Reynolds number. We see that for each $R e$ the mixing has resulted in the profile $L(r)$ becoming flat with value 0 from the center $r=0$ to some position beyond $r_{+}$, the limit of the linearly unstable region, except for some minor fluctuations due to residual action of the decaying perturbation vortices. We could look at these profiles at a much later time when the perturbation vortices have more or less completely decayed, but by that time, the effects of viscosity will completely dominate and the effect of centrifugal instability will no longer be evident. 
Note that in Fig. 10a, the higher the value of $R e$ the further out the region of $L(r) \approx 0$ extends. Also note that for each $R e$ there is a transition region where $L(r)$ climbs from $L(r) \approx 0$ to join the curve corresponding to the unperturbed vortex, the thin line curve that we will denote $L_{0}(r)$. Finally, note that in that transition region, the profile of $L(r)$ becomes steeper as Re increases. Putting these, and other similar, observations together, Kloosterziel, Carnevale and Orlandi (2007)[8] formulated a prediction scheme for the saturation of centrifugal instability in the limit of $R e \rightarrow \infty$. In this limit they predict that mixing would be 'perfect' in the sense that $L$ would be mixed to a uniform value in a mixing region within and around the initial linearly unstable region. Beyond the mixing region, they predict that the final curve $L(r)$ would coincide with the initial unperturbed profile $L_{0}(r)$. Jumps in $L(r)$ where the mixing region joins $L_{0}(r)$ are permitted as long as the jumps are stable, that is as long as the sense of the jump satisfies the stability condition $\Delta L^{2}>0$ in going from $r$ smaller than the jump position to $r$ larger than the jump position. Although conservation of $L$ on particles can be lost due to viscous effects even in the limit of $R e \rightarrow \infty$, Kloosterziel, Carnevale and Orlandi (2007)[8] claimed that for any unstable profile, the limits of the mixing region can be predicted from the conservation of total absolute angular momentum, which is valid at all $R e$. Thus, for the case of the unperturbed profile shown in Fig. 10a (based on formula (1) with $\alpha=3$ and $R o=-4$ ), the prediction would be that in the limit $R e \rightarrow \infty$, the final profile would be
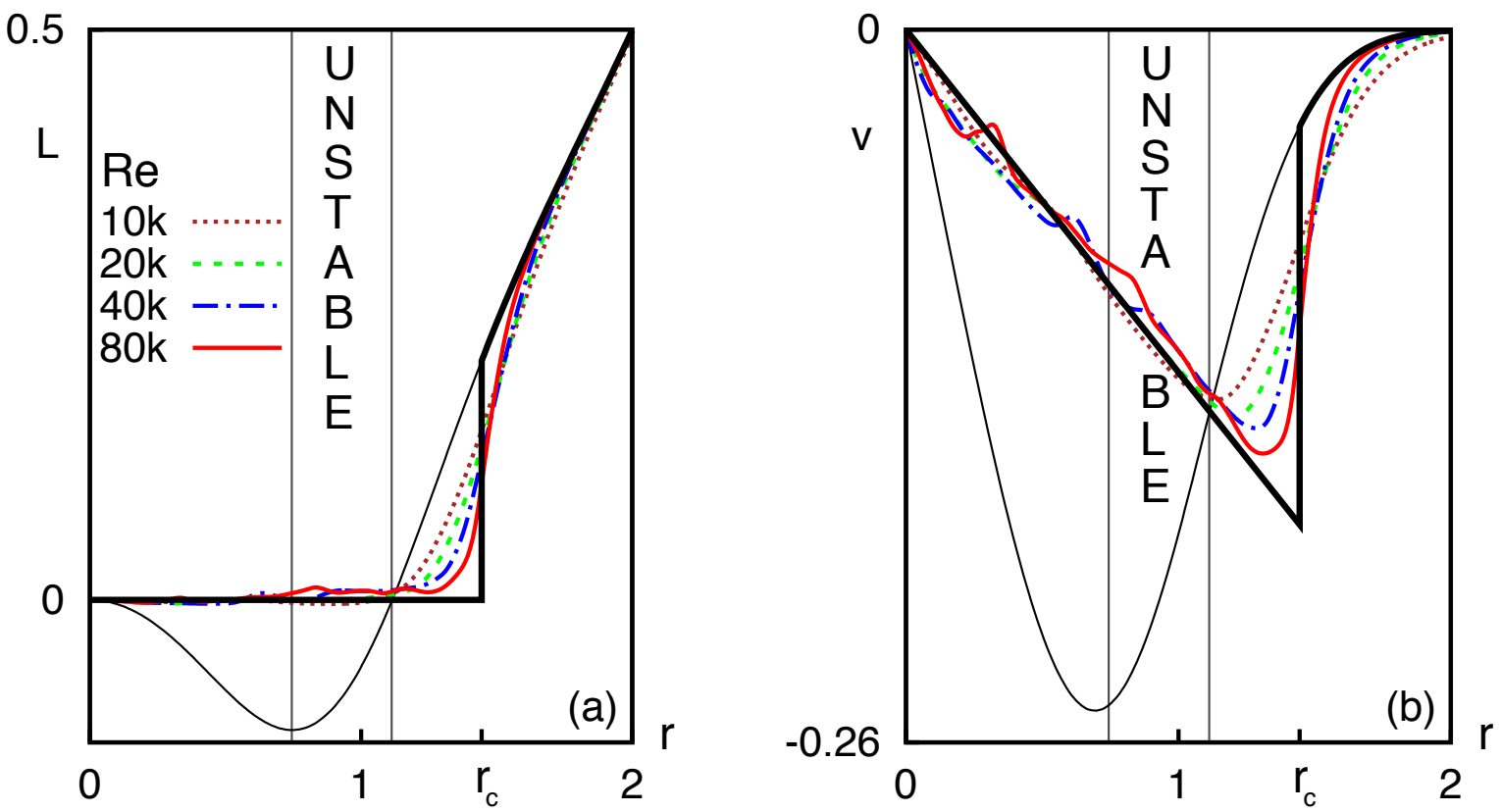

Figure 10. Profiles of absolute angular momentum $L=r(v+\Omega r)$ and velocity $v$, nondimensionalized as discussed in the text, for several values of $R e$. The thin curve is the initial condition corresponding to the anticyclonic vortex with $R o=-4$ and $\alpha=3$. The thin vertical lines at $r_{-}=0.745$ and $r_{+}=1.11$ mark the limits of the linearly unstable region. The thick colored curves correspond to time $t=300$. The thick black curve is our $R e \rightarrow \infty$ prediction. The critical radius $r_{c}=1.45$ is calculated from formula (11). (a) $L$; (b) $v$.

$$
L(r)= \begin{cases}0 & 0<r<r_{c} \\ L_{0}(r) & r_{c}<r .\end{cases}
$$

The position $r_{c}$ is at the edge of the mixing region. It is determined by the condition of total angular momentum conservation. For the current example, the difference between the unperturbed $L$ and the the final $L$ in the mixing region is just $L_{0}$. Thus, for the total change in $L$ to vanish, we must have

$$
\int_{0}^{r_{c}} L_{0}(r) r d r=0
$$


where

$$
L_{0}(r)=r(V(r)+\Omega r)
$$

and $V(r)$ is the unperturbed profile (1). The 'element' of integration is $r d r$ since we are concerned with the total $L$ in the $3 \mathrm{D}$ vortex and we are using cylindrical coordinates.

This formula (12) determines $r_{c}$. The result is that the total volume integrated negative $L$ in the range $0<r<r_{+}$ will be mixed homogeneously with the positive $L$ from the range $r_{+}<r<r_{c}$ to the value 0 in the whole mixing range $0<r<r_{c}$. The essence of this prediction is that the mixing is thorough and uniform and the mixing region extends only as far as it must in order to bring in sufficient positive $L$ to mix with the negative $L$ in the core. The prediction (11) is drawn as a thick black curve in Fig. 10a.

Since $L(r)=r(v(r)+\Omega r)$, the prediction for the velocity profile is

$$
v(r)= \begin{cases}-\Omega r & 0<r<r_{c} \\ V(r) & r>r_{c} .\end{cases}
$$

Remarkably, this predicts that the entire core $\left(r<r_{c}\right)$ of the equilibrated vortex will be in solid body rotation with angular velocity exactly the opposite of that of the tank. This means that in the lab frame of reference, the core will be stationary!

The prediction for the equilibrium velocity is shown as the thick black curve in Fig. 10b. As Re increases, the curves from the simulations extend further and further along the part of the curve indicating solid body rotation, from $r=0$ to $r=r_{c}$, and the curves come closer to matching the limiting $R e=\infty$ jump.
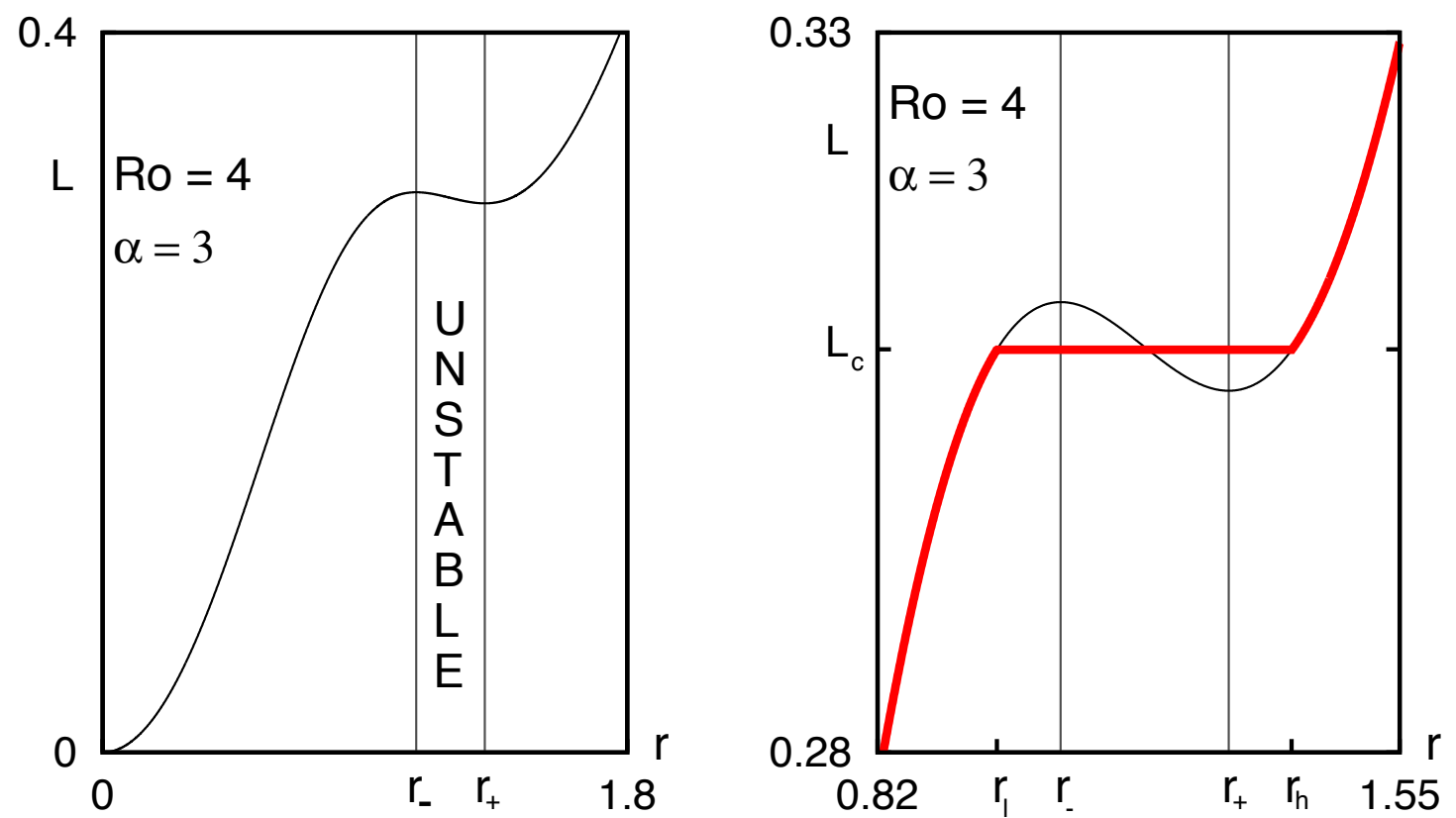

Figure 11. Profiles of absolute angular momentum $L=r(v+\Omega r)$ nondimensionalized as discussed in the text, for an unstable cyclone $($ Ro $=+4$ and $\alpha=3$ ). The thin curve is the initial condition. The thin vertical lines at $r_{-}=1.08$ and $r_{+}=1.31$ mark the limits of the linearly unstable region. (a) The initial profile for $L$. (b) Expanded view of the profile in and around the instability region. The thick red curve is our prediction for $R e \rightarrow \infty$. The limits on the mixing region $r_{l}=0.987$ and $r_{h}=1.40$, and the level of mixing $L_{c}=0.308$ are calculated from formula (15).

\subsection{Nonlinear equilibration: Cyclones}

As determined by formulas (8) and (9), a cyclone can also be centrifugally unstable if sufficiently strong. In the last section, we considered an anticyclone with $R o=-4$ and $\alpha=3$. For comparison, we now briefly consider the same 


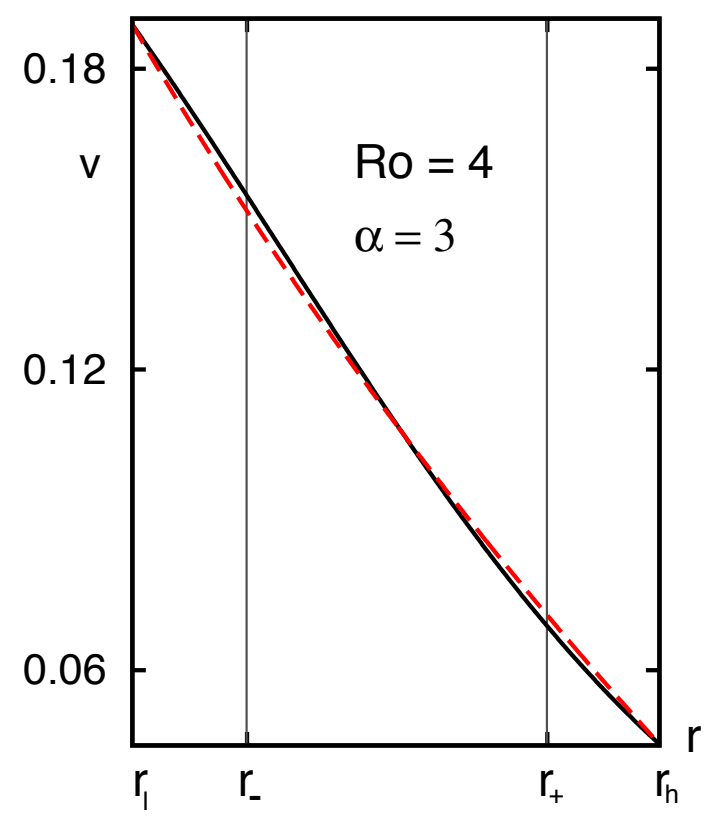

Figure 12. Comparison of the initial profile of velocity with the prediction for the unstable cyclone $(R o=+4$ and $\alpha=3)$ whose $L$ profile is shown in 11. The curves are nondimensionalized as discussed in the text. Only the mixing range is shown since the curves are identical outside that range. The solid black curve is the initial condition and the dashed curve is the prediction for $R e \rightarrow \infty$. The thin vertical lines at $r_{-}=1.08$ and $r_{+}=1.31$ mark the limits of the linearly unstable region. The limits on the mixing region are $r_{l}=0.987$ and $r_{h}=1.40$. The maximum steepness of the red curve is $0.6 \%$ less than that of the black curve, so equilibration for the cyclone diminishes the velocity gradient at the boundary of the core region.

vortex with velocity reversed, that is a cyclone with $R o=+4$ and $\alpha=3$. The profile of absolute angular momentum $L(r)$ for this vortex is shown in Fig. 11a. We see that there is a narrow instability region, between $r_{-}$and $r_{+}$, where the magnitude of $L$ decreases. Note how different the profile is from the profile of $L$ for the anticyclone shown in Fig. 8. Unlike the anticyclonic case, for the cyclone $L>0$ for all $r$, and thus there can be no mixing of positive and negative angular momentum during the instability. Nevertheless, the instability unfolds very much like in the case for the anticyclone: a stack of vortex rings form during the linear phase; dipolar heads and tails form as nonlinear effects become important; by advection the dipoles propagate out of the region of linear instability; the flow becomes chaotic due to the nonlinear interaction between the dipoles; and finally mixing of $L$ brings about a new stable profile Kloosterxiel et al.(2007a)[8]. The big difference, though, is that for the anticyclone, the dipoles penetrated deep into the core of the vortex, all the way to its center and equilibration involved most of the vortex. For the cyclone, on the other hand, most of the action is confined to the area in and immediately around the narrow instability region. The core of the cyclone is not involved at all.

The same prediction scheme discussed in subsection 3.1 can be applied here with minor adjustments. In figure $11 \mathrm{~b}$, we have zoomed in on the profile of $L$ in the instability region. There we see that complete mixing to uniform $L$ in the region in and around the instability region would result in a stable profile. Such an equilibrium is represented as the thick line overlying the thin line representing the initial profile of $L$. During the equilibration, the 'excess' angular momentum lying above the horizontal line at the level marked $L_{c}$ and near $r_{-}$will have uniformly mixed with the 'deficit' angular momentum lying below this line in the area around $r_{+}$. Conservation of total angular momentum tells us where the limits, $r_{l}$ and $r_{h}$, of the uniformly mixed region must be. The formula then for determining $L_{c}$ and these limits is

$$
\int_{r_{l}}^{r_{h}}\left(L_{0}(r)-L_{c}\right) r d r=0 \quad \text { where again } \quad L_{0}(r)=r(V(r)+\Omega r)
$$


with $V(r)$ the profile (1) and where $r_{l}$ and $r_{h}$ satisfy the auxiliary matching conditions

$$
L_{c}=L_{0}\left(r_{l}\right)=L_{0}\left(r_{h}\right) \text {. }
$$

Note that there is no need for a jump in this equilibrium, as there was in the anticyclonic case. Here, mixing to a region of uniform $L$ to achieve stability can be accomplished without the introduction of a jump. Examples of simulations showing that this prediction does very well for a variety of cyclones have been given in Kloosterziel et al.(2007a)[8].

To consider what effect the centrifugal instability may have on the subsequent barotropic instability, it is necessary to examine the change produced in the velocity profile. In Fig. 12 we see the initial azimuthal velocity of the vortex (black solid curve) in the region where mixing will occur and the predicted velocity (dashed curve). The two lines are not very different, in contrast to the anticyclonic case where the equilibrated velocity profile is dramatically different from the initial profile. For the barotropic instability, an important question is the steepness of the profile, that is the horizontal gradient of the vorticity, at the outer boundary of the core region. We saw that for the anticyclone, the centrifugal instability made the velocity much steeper for high values of $R e$, and infinitely steep, according to the prediction, in the limit $R e \rightarrow \infty$. If we compare the initial and equilibrated profiles for the cyclone, we find that the predicted profile would be less steep (by $0.6 \%$ ) than the initial profile. That would make barotropic instability weaker, not stronger. If we think of $\alpha$ as a measure of steepness, and look at figure 6 , we see that at $\alpha=3$, which is the value for our cyclone and anticyclone examples, only mode $m=2$ can grow, the mode that corresponds to tripole production. Since the centrifugal instability makes the velocity profile less steep in the cyclonic case, one could only expect a tripole from the barotropic instability. On the other hand, by increasing the steepness of the velocity profile for the anticyclone, which would be analogous to increasing $\alpha$ in Fig. 6, we would expect growth in mode 3 (and perhaps higher) as well as mode 2, leading to structures more complicated than the tripole, such as the pair of dipoles shown in Fig. 3.

\section{Predicting the outcome of the full 3D evolution}

With the understanding developed for pure barotropic and pure centrifugal instability and how they separately equilibrate, we are now in a position to use this knowledge to try to predict the outcome of their combined effect on the evolution in the fully 3D flow. The idea is based on the observations in the laboratory experiments that the centrifugal instability is much faster than the barotropic instability Kloosterziel and van Heijst (1991)[1]. In such a circumstance, we can imagine the centrifugal instability rapidly mixing absolute angular momentum and creating a new distribution of $L$ before the barotropic instability has proceeded very far. We could then attempt a prediction by supposing that centrifugal instability occurs and saturates nonlinearly, producing a new state $L=L(r)$ even before barotropic instability begins. For the new state $L(r)$, we can take the inviscid prediction given in section 3 . There are no a priori predictions possible for the barotropic instability, but we can easily calculate that evolution using numerical simulation of the pure 2D flow. In this second stage of the prediction, we can use a simulation at finite $R e$ to best match the results of the rotating tank experiments and our fully 3D numerical simulations, which are necessarily at finite $R e$. This is the basic scheme as first described by Carnevale et al.(2011)[5]. In what follows, we shall explore the validity of the predictions so made and predict results for the evolution of vortices in a wide range in the parameter space determined by $\alpha, R o$ and $R e$. Since the evolution of cyclones is mostly determined by the barotropic instability at values of $\alpha$ and Ro typical in the laboratory experiments, we will restrict ourselves from this point on to examining the more complicated behavior of anticyclones.

\subsection{Testing the prediction when centrifugal instability is much faster than barotropic: example $\alpha=1.8$}

Recall that Fig. 6 shows that there is no barotropic instability for vortices with $\alpha \lesssim 1.85$. A centrifugally unstable vortex with $\alpha \lesssim 1.85$ should then be a case where we would expect our prediction scheme to produce the best results. No barotropic instability would occur at first, and it would only be possible if the centrifugal instability steepens velocity gradients sufficiently to produce barotropic instability. In this subsection, we will look in detail at the case $\alpha=1.8$.

Although the barotropic instability is independent of the Rossby number $R o$, the centrifugal instability is not. For an anticyclone, we must have $R o<-1$ for instability as discussed in section 3. In Fig. 13, we show the evolution of the azimuthal vorticity field $\omega_{\theta}$ for the case $\alpha=1.8, R o=-2.35$ and $R e=15 \mathrm{k}$ (i.e. $R e=15,000$ ). This is a 3D image 


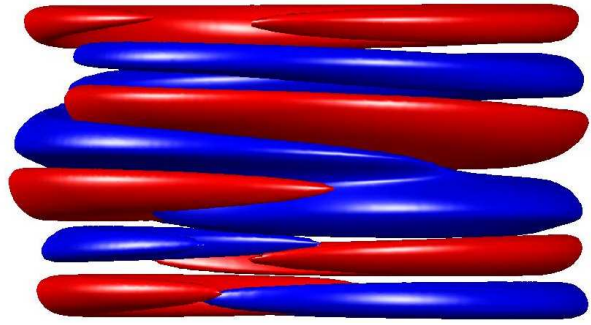

(a) $t=90$

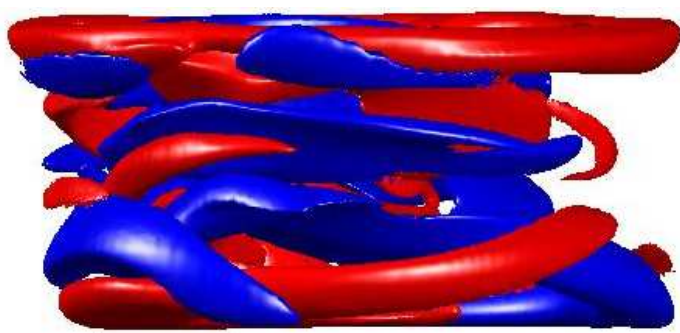

(c) 140

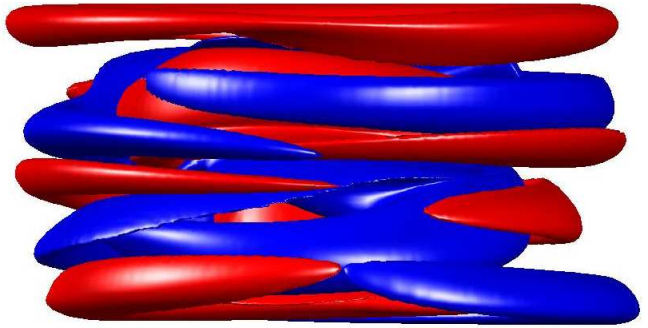

(b) 120

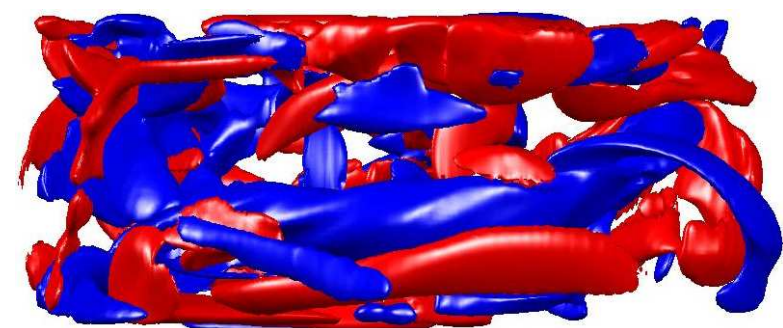

(d) 190

Figure 13. The evolution of the azimuthal component of the vorticity field $\omega_{\theta}$ in the case $\alpha=1.8, R o=-2.35$ and $R e=15 \mathrm{k}$. Red/blue corresponds to positive/negative $\omega_{\theta}$. The view is from the side, that is along a line perpendicular to the axis of the primary or basic vortex. The magnitude of $\omega_{\theta}$ on the plotted isosurfaces increases in time from $3.2 \times 10^{-3}$ at $t=90$ to $3.3 \times 10^{-1}$ at $t=190$. (Reproduced from Carnevale et al.(2011)[5] with permission from Cambridge University Press)

rendering of the field that can be compared to the 2D cross sections shown in Fig. 9 for the data from the azimuthally symmetric simulation. There are important similarities and difference between these two figures worthy of note. First of all, both figures show an early stage in which the dominant feature is the stack of vortex rings created by the linear phase of the instability. The main difference at this, and indeed at all phases of the instability, is the that the 3D flow is not azimuthally symmetric. In Fig. 13b, dipolar pairing is evident in the outer field. This is similar to that found in the azimuthally symmetric simulation. We see here that this stage also occurs in the 3D flow despite strong asymmetries. The strongly non-linear phase shown in $9 \mathrm{c}$ was remarkable for the complicated patterns formed by the interactions of the migrating dipolar heads and tails. Here in Fig. 13d, we see that with the additional freedom of fully 3D evolution, the chaotic intertwining of the vortex structures becomes even more complicated.

Interestingly, even though azimuthal symmetry is lost in the 3D flow, the net effect of the development of the dipolar structures is to change the vertically averaged profile much as in the azimuthally symmetric simulations, creating an enhanced velocity gradient in the outer core region Carnevale et al.(2011)[5]. After the time shown in Fig. 13d $(\mathrm{t}=190)$, the flow becomes increasingly vertically uniform, and a barotropic instability becomes evident. The result of the barotropic instability is best visualized by taking the vertical average of the vertical vorticity $\omega_{z}$. The evolution of this averaged $\omega_{z}$ is illustrated in Fig. 14. Here we see the flow evolves from a monopolar structure into a tripole with a core of anticyclonic vorticity and two cyclonic satellites, as in the case of the laboratory experiment shown in Fig. 4. The images suggest that mainly the barotropic instability results in azimuthal mode $\mathrm{m}=2$ growth (see formula (4) and Fig. 6). It seems reasonable to conclude that the centrifugal instability phase of the 3D evolution produced sufficient steepening of the velocity profile, in this initially barotropically stable vortex, to force significant growth in the amplitude of azimuthal mode $\mathrm{m}=2$, but not in higher order modes. A detailed analysis of the modal growth is given in Carnevale et al. [5]. There it found that, in the barotropic phase of the flow, after time $t=300$, the amplitude in mode 3 is insignificant and the amplitude of mode $m=4$ is less than about $15 \%$ of that of mode 2 . 


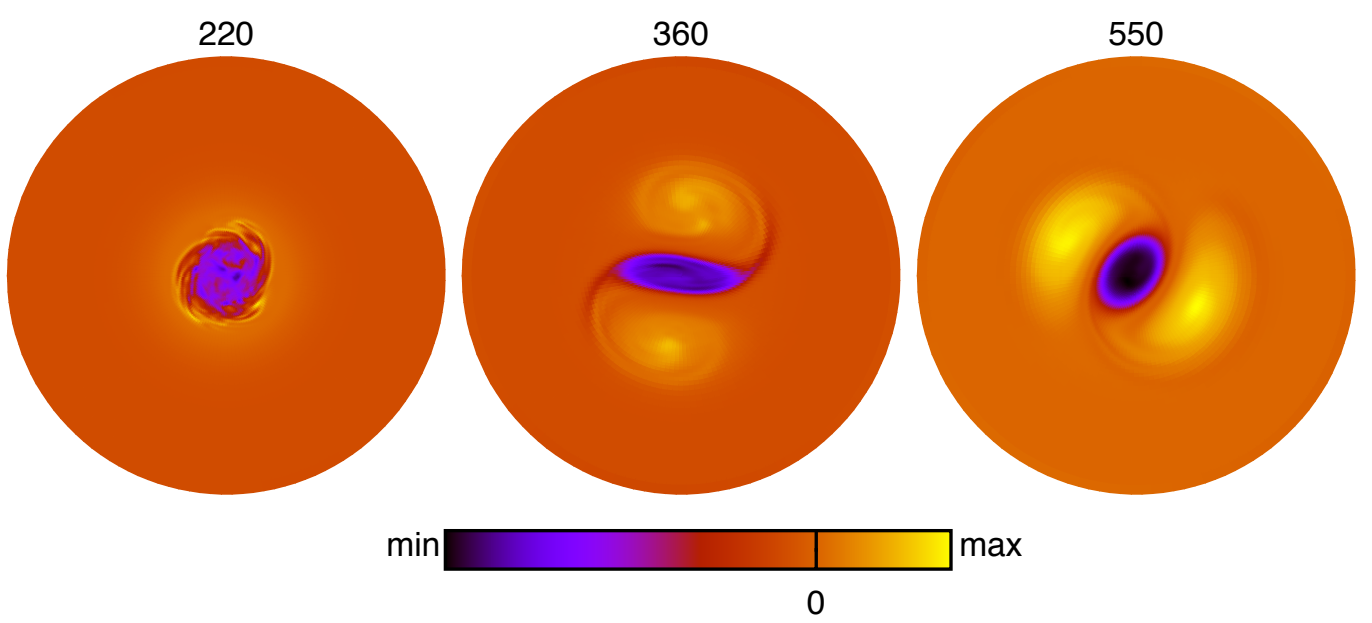

Figure 14. $\omega_{z}$ averaged over depth $z$ for the vortex with $\alpha=1.8, R o=-2.35$ in a 3D numerical simulation with $R e=15 \mathrm{k}$. The minimum and maximum of $\omega_{z}$ for each panel are: $t=220(\min =-2, \max =1), t=360(-1.2,0.6)$ and $t=550(-1.2,0.4)$. (Reproduced from Carnevale $e t$ al.(2011)[5] with permission from Cambridge University Press. Note that in Carnevale et al.(2011)[5], these images were inverted by mistake. The presentation here is correct.)

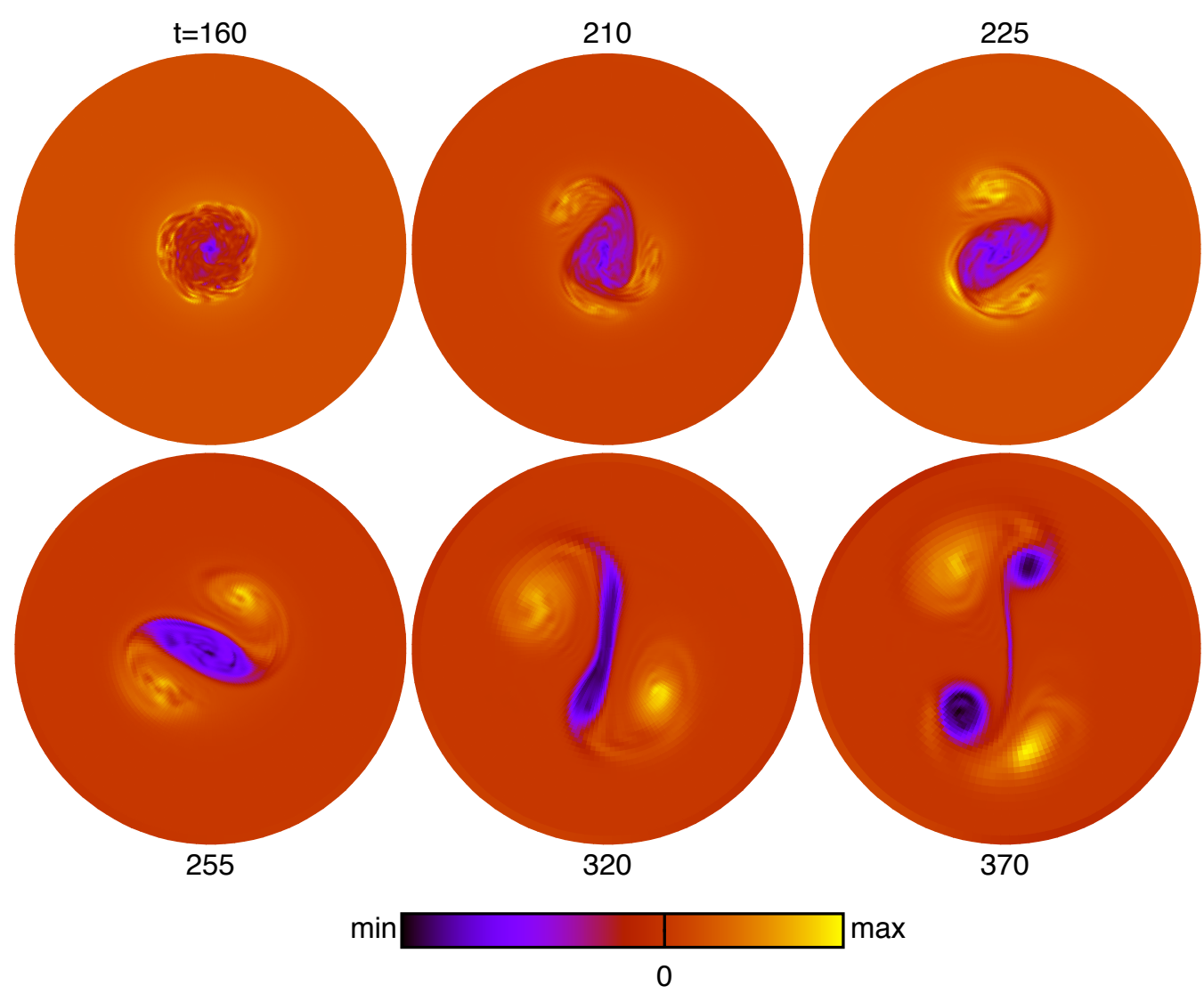

Figure 15. $\omega_{z}$ averaged over depth $z$ for the vortex with $\alpha=1.8, R o=-3$ in a numerical simulation with $R e=15 \mathrm{k}$. The minimum and maximum of $\omega_{z}$ for each panel are: $t=160(-4,2), t=210(-2.5,1.5), t=225(-2 ., 1),. t=255(-1.5,1),. t=320(-1.2,0.8)$ and $t=370(-1.2,0.8)$. (Reproduced from Carnevale et al.(2011)[5] with permission from Cambridge University Press) 

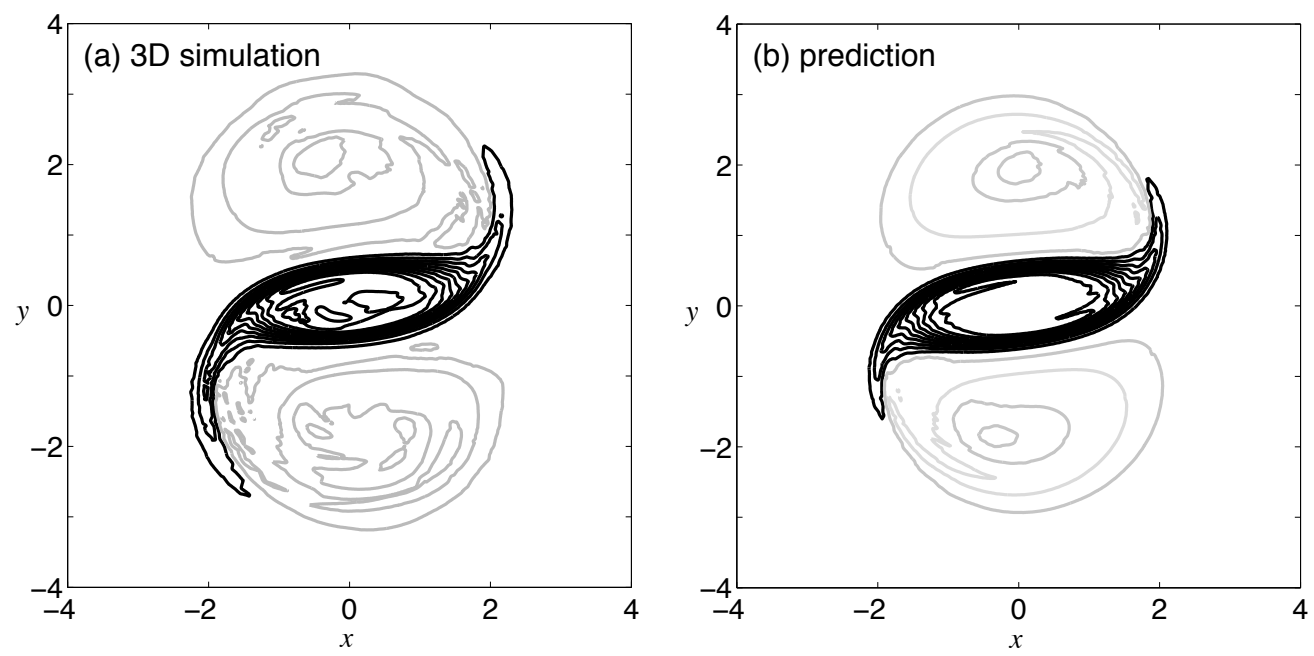

Figure 16. Comparison of the results of the three-dimensional simulation with the prediction. (a) Vorticity field at mid depth at time $t=380$ in the fully three-dimensional simulation. (b) Predicted vorticity field at time 330 from the two-dimensional simulation of the barotropic part of the prediction. The isolevels increase from -0.5 to 0.3 with increments of 0.04 . Black/gray contours represent negative/positive isolevels. ( $\alpha=1.8, R o=-2.35, R e=15 \mathrm{k}$ ) (Reproduced from Carnevale et al.(2011)[5] with permission from Cambridge University Press)

With $\alpha$ fixed at 1.8 (barotropically stable), we then decreased $R o$ from -2.35 , the value used in the simulation illustrated in Fig. 13 and Fig. 14, to Ro $=-3$. Images of the 3D structure of $\omega_{\theta}$ in this case are very similar to those shown in Fig. 13. On the other hand, the resulting evolution of the vertically averages $\omega_{z}$ proves to be very different. This is shown in Fig. 15. The flow evolves from a monopolar vortex at time $t=160$ into a structure showing a significant component of mode 3 asymmetry by $t=210$. This structure, a quadrapole, has an anticyclonic core and 3 cyclonic satellites. As the evolution proceeds, two of the satellites merge into one, the common instability path for high order multipoles (see Carnevale and Kloosterziel, 1994[4]), and by $t=255$, the structure has become a tripole. But this tripole is not stable because in addition to mode 2, which is primarily associated with the tripolar shape, higher order modes are present and growing. The result is that the tripole splits into a pair of dipoles that propagate away from each other. Analysis of the azimuthal modal content of the barotropic component of the flow in Carnevale et al. (2011)[5] showed that modes 1,2,3 and 4 were all significant, with the amplitude of modes 2 and 4 being two or more times as large as that of modes 1 and 3 .

We next show direct comparisons between the 3D simulation results and our prediction. Fig. 16 shows the result in the $\alpha=1.8, R o=-2.35$ case. In panel (a), we see the vertical vorticity field at mid depth (not an average) in the 3D simulation at time $t=380$. In panel (b), we present the vertical vorticity from the pure 2D simulation of our prediction scheme at $t=330$. We cannot expect the times to match well since, in the prediction scheme, we replace the entire centrifugal instability phase of the flow with our analytic prediction for the adjusted $L(r)$. Here we have chosen to compare the prediction and the 3D simulation at a time when similar structures have resulted. Note that the azimuthal orientation of the two figures is not significant since it relies entirely on the nature of the small scale random perturbation to these flows. Here we have just oriented both structures the same way to facilitate comparison. The structures are remarkably similar not just in that they are both tripoles, but also in the structure of the component vortices.

Fig. 17 shows a similar comparison for the $\alpha=1.8, R o=-3$ case. Again the comparison is rather remarkable, especially when we note that panel (a) is the cross section of the $3 \mathrm{D}$ field and not a vertical average.

\subsection{Regime diagram: $\alpha=1.8$}

Another test of the skill of our prediction scheme is to create regime diagrams in the parameter space $(\alpha, R o$, $R e$ ) that will show in which parameter ranges one should expect to find tripoles or double dipoles as a result of the evolution of a centrifugally unstable vortex. For $\alpha=1.8$, such a regime diagram is shown in Fig. 18. The solid grey curve shows the boundary between the regions in $(R e, R o)$ in which a single monopole or a single tripole result 

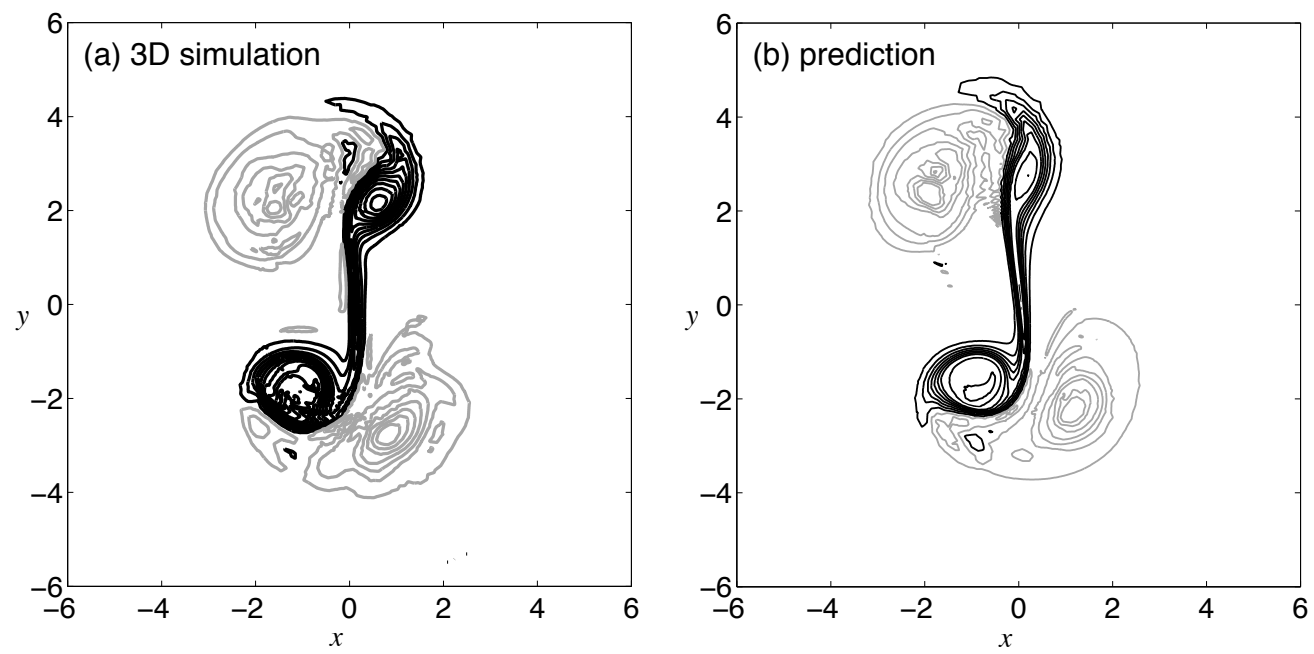

Figure 17. Comparison of the results of the three-dimensional simulation with the prediction. (a) Vorticity field at mid depth at time $t=360$ in the fully three-dimensional simulation. (b) Predicted vorticity field at time 240 from the two-dimensional simulation of the barotropic part of the prediction. The isolevels increase from -0.4 to 0.2 with increments of 0.03 . Black/gray contours represent negative/positive isolevels. ( $\alpha=1.8, R o=-3.0, R e=15 \mathrm{k})$ (Reproduced from Carnevale et al.(2011)[5] with permission from Cambridge University Press)

from the evolution of a full 3D simulation. Similarly the solid black curve shows the boundary between the regions in which we find a single tripole or a pair of dipoles as the results of full 3D simulations. The error bars on those curves indicate the sampling error, that is for each $R e$ we have run a series of 3D simulations with different values of $R o$, and the error bar shows the distance to the simulations on either side of the solid curve that determined the position of the curve. The solid curves may be compared with the dashed curves of like color that were calculated via our prediction scheme.

Note that there is a systematic trend to these curves, the value of $R o$ increases monotonically with $R e$. This should be related to the competing effects of centrifugal instability to increase the steepness of the velocity profile and of viscosity to smooth it. The more viscous the flow, the more negative Ro needs to be to give a sufficiently strong fall off of velocity outside the core region to produce a sufficiently vigorous barotropic instability to see the higher mode structures develop.

The comparison between the solid and dashed curves reveals that the prediction scheme is more reliable for higher $R e$ than for low. For both boundaries this result is expected since the centrifugal instability part of the prediction scheme is based on the $R e \rightarrow \infty$ limit, and so the overall prediction should be better the higher the value of $R e$. One of the important physical insights that we can gain from this diagram is related to the fact that the tripole regime is relatively narrow compared to that of monopoles (which extends down to $R o=0$ ) and double-dipoles. This is likely the reason why very few cases of tripole formation from anticyclonic vortices were observed in the laboratory experiments.

\subsection{Testing the prediction for a range of $\alpha$}

As discussed above, the choice $\alpha<1.85$ is optimal for our prediction scheme since this scheme is based on centrifugal instability being faster than barotropic instability and for $\alpha<1.85$ the barotropic instability growth rate is zero. But does our prediction scheme work well when the growth rates of centrifugal and barotropic instability are comparable?

It is seen with Fig. 6 that the non-dimensional exponential growth rate $\gamma$ for barotropic instability for $\alpha=3$ is approximately 0.077 . The growth rate for centrifugal instability at this $\alpha$ has been determined by axisymmetric simulations Carnevale et al.(2011)[5]. It was found that as Ro decreases from -1 to -2.2, the centrifugal instability growth rate, for $R e=15 \mathrm{k}$, increases from 0 to 0.073 . Thus for a vortex with $\alpha=3$ and $R o=-2.2$ we can expect comparable growth rates for both centrifugal and barotropic instabilities. In Fig. 19, we show the tripole to doubledipole regime boundaries from the full 3D simulations (solid black line) and our prediction scheme (dashed grey line). 


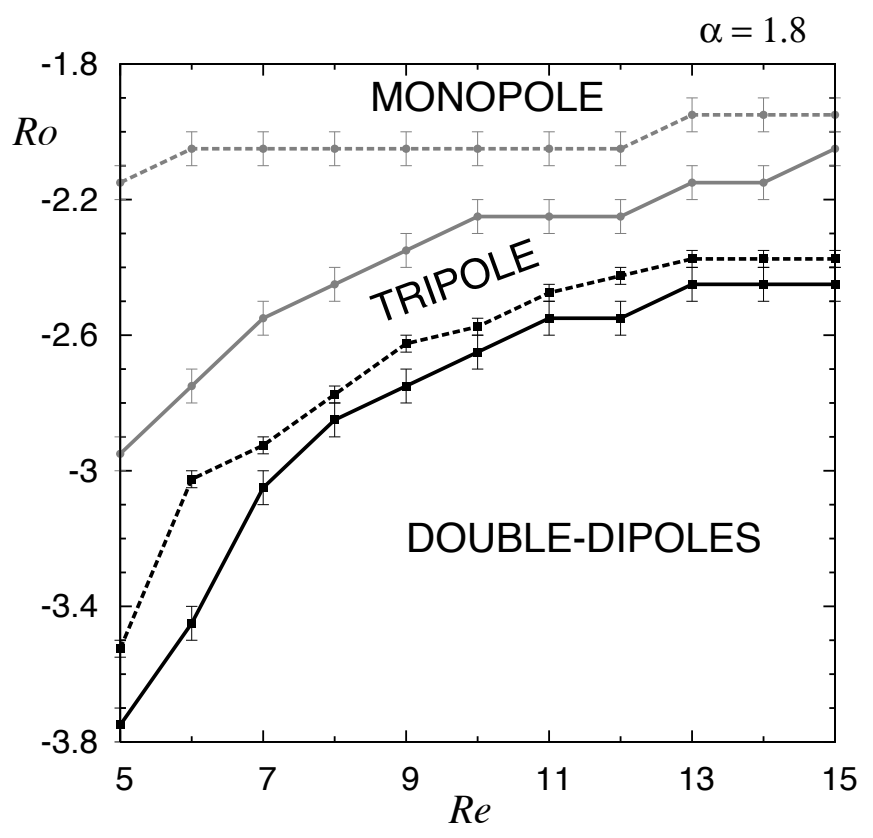

Figure 18. Regime diagram for the case with $\alpha=1.8$, in which the centrifugal instability is initially faster than the barotropic instability. The thick solid black curve is the boundary between tripoles and double-dipoles resulting from 3D simulations. The dashed black curve is the prediction for this boundary. The thick solid gray curve is the boundary between monopoles and tripoles resulting from 3D simulations. The dashed gray curve is the prediction for this boundary. The sampling error is indicated by the error bars. Re is given in units of 1000. (Reproduced from Carnevale $e t$ al.(2011)[5] with permission from Cambridge University Press)

The graph covers the range of $\alpha$ from $\alpha=1.8$ where the barotropic growth rate is 0 to $\alpha=3$ where the barotropic growth rate it 0.077 . Only the double-dipole region has been marked explicitly. Just above the double-dipole to tripole boundary, only tripoles form. However, on the low $\alpha$ side of the graph, there is a region of monopole formation for $R o$ sufficiently close to -1 (the limit of centrifugal instability). On the high $\alpha$ end of the figure, in particular at $\alpha=3$, there is no monopole regime no matter how small $|R o|$.

Note that the difference between the prediction and the 3D simulation result increases as $\alpha$ increases. This makes sense since our prediction scheme is expected to be best when the centrifugal instability is faster than the barotropic instability, giving time for complete mixing of absolute angular momentum before barotropic instability can proceed very far. For high $\alpha$, at best we can say that both instabilities are proceeding at the same time and the prediction performs least well there. Nevertheless, it is gratifying to see that the prediction scheme appears to have skill at all values of $\alpha$ plotted.

\section{Discussion}

In this article, we have reviewed the work that we have performed on the way centrifugal and barotropic instabilities unfold in initially barotropic vortices. A scheme that is rather skillful in predicting the end-state of the evolution of centrifugally unstable barotropic vortices in fully 3D flow has been proposed and tested rather successfully. Similar results can be demonstrated in density stratified flow as well Kloosterziel et al.(2007a)[8].

Centrifugal instability is a type of inertial instability specific to vortices. In other work, we have shown how a variant of our prediction scheme, based on absolute linear momentum mixing, can very accurately predict the unfolding of pure, that is independent of along-flow variation, inertial instability in barotropic planar currents (Kloosterziel et al.2007b[27]). In Carnevale et al.(2013)[28], we showed that a prediction scheme based on our mixing prescription for inertial instability combined with 2D simulation could predict the characteristics and statistics of the vortices that result from the breakup of an initially planar barotropic current in fully 3D flow. See also the interesting work of Plougonven and Zeitlin (2009)[29] and Bouchut et al.(2011)[30] on this topic. 


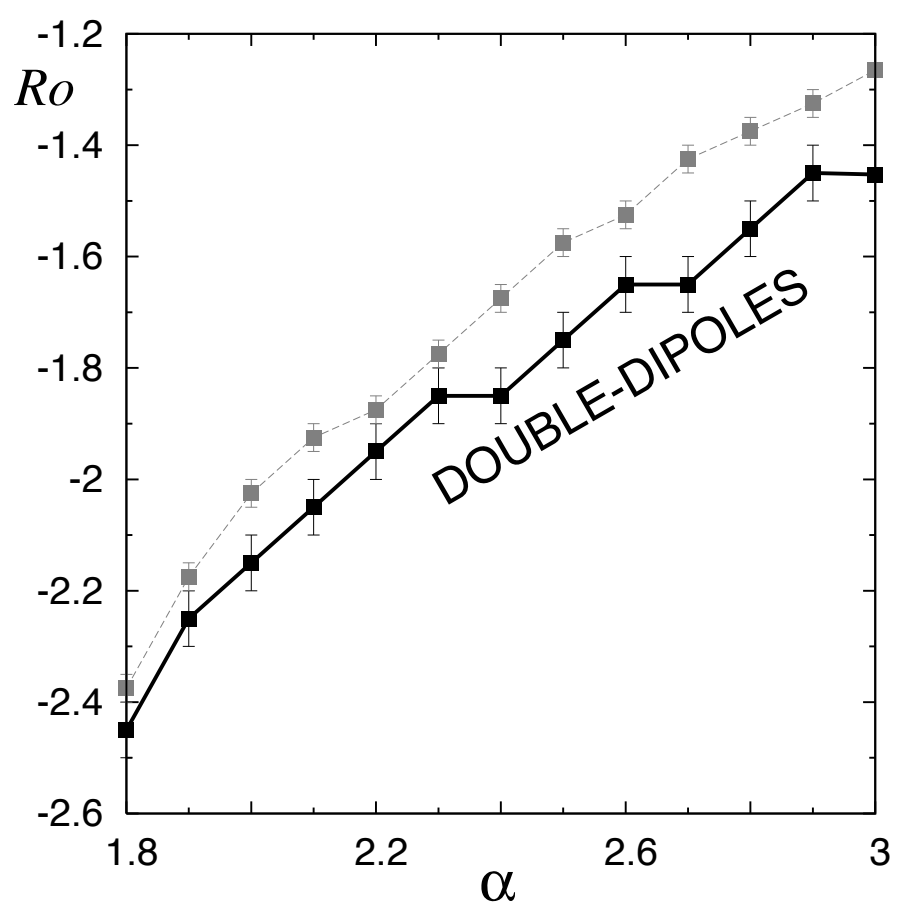

Figure 19. The boundary between double-dipole and tripole regimes as predicted (thin dashed grey curve) and from results of fully 3D numerical simulations (thick solid black curve). (Reproduced from Carnevale et al.(2011)[5] with permission from Cambridge University Press)

All of these results were obtained for constant background rotation $\Omega$. This restriction was relaxed in our recent work Kloosterziel et al.(2015)[31] where we extended our results to include the effect of variation of $\Omega$ with latitude, the $\beta$-effect. In that work we considered jet flow in the equatorial regions using a standard model that considers only the component of rotation perpendicular to the surface of the earth. In future work, we will show how the neglected component of rotation, that parallel to the surface of the earth plays a crucial role in determining the kind of momentum mixing that will occur near the equator when inertial instability occurs.

Finally, we will mention that we are also working on extending this research to consider baroclinic vortices and currents, that is those whose velocity varies with depth, in stratified flow. In that problem, mixing momentum becomes far more complicated than in the barotropic case. Stationary baroclinic flows have iso-density surfaces (isopycnals) that are not flat. Momentum mixing occurs primarily in between the isopycnals. However, it is difficult to predict the mixing because the shape of the isopycnals changes as the mixing proceeds. It is a challenge to predict both the extent of the mixing and the change of the shape of the isopycnals since they are completely interdependent.

GFC acknowledges support from National Science Foundation grant OCE 11-29059. RCK acknowledges support from National Science Foundation grant OCE 10-32256. The authors have benefited greatly in this work from the help and interaction with many others. In particular, we would like to thank Gert-Jan van Heijst and Daan van Sommeren for their contributions to some of the main papers on which this review is based.

\section{References}

[1] R. C. Kloosterziel, G. van Heijst, An experimental study of unstable barotropic vortices in a rotating fluid, J. Fluid. Mech. 223 (1991) 1-24.

[2] G. J. F. van Heijst, R. C. Kloosterziel, Tripolar vortices in a rotating fluid, Nature 338 (1989) 569-571.

[3] G. J. F. van Heijst, R. C. Kloosterziel, C. W. M. Williams, Laboratory experiments on the tripolar vortex in a rotating fluid, J. Fluid. Mech. 225 (1991) 301-331.

[4] G. F. Carnevale, R. C. Kloosterziel, Emergence and evolution of triangular vortices, J. Fluid. Mech. 259 (1994) 305-331.

[5] G. F. Carnevale, R. C. Kloosterziel, P. Orlandi, D. D. J. A. van Sommeren, Predicting the aftermath of vortex breakup in rotating flow, J. Fluid Mech. 669 (2011) $90-119$. 
[6] P. Orlandi, G. F. Carnevale, Evolution of isolated vortices in a rotating fluid of finite depth, J. Fluid. Mech. 381 (1999) $239-269$.

[7] G. F. Carnevale, M. Briscolini, R. C. Kloosterziel, G. K. Vallis, Three-dimensionally perturbed vortex tubes in a rotating flow, J. Fluid Mech. 341 (1997) $127-163$.

[8] R. C. Kloosterziel, G. F. Carnevale, P. Orlandi, Inertial instability in rotating and stratified fluids: barotropic vortices, J. Fluid. Mech. 583 (2007) 379-412.

[9] L. Rayleigh, On the stability, or instability, of certain fluid motions, Proc. London Math. Soc. 11 (1880) 57 - 70.

[10] P. G. Drazin, W. H. Reid, Hydrodynamic Stability, Cambridge University Press, 1981.

[11] M. E. Stern, Horizontal entrainment and detrainment in large-scale eddies, J. Phys. Oceanogr. 17 (1987) $1688-1695$.

[12] G. R. Flierl, On the instability of geostrophic vortices, J. Fluid. Mech. 197 (1988) 349-388.

[13] R. C. Kloosterziel, G. F. Carnevale, Formal stability of circular vortices, J. Fluid. Mech. 242 (1992) $249-278$.

[14] X. Carton, J. C. McWilliams, Barotropic and baroclinic instabilities of axisymmetric vortices in a quasi-geostrophic model, in: J. C. J. Nihoul, B. M. Jamart (Eds.), Mesoscale/Synotopic Coherent Structures in Geophysical Turbulence, Elsevier, New York, 1989 , pp. 225 - 244.

[15] X. J. Carton, G. R. Fierl, L. M. Polvani, The generation of tripoles from unstable axisymmetric isolated vortex structures, Europhys. Lett. 9 (1989) 339-344.

[16] P. Orlandi, G. J. F. van Heijst, Numerical simulation of tripolar vortices in 2d flow, Fluid Dyn. Res. 9 (1992) $179-206$.

[17] X. J. Carton, B. Legras, The life-cycle of tripoles in two-diemsional incompressible flows, J. Fluid Mech. 267 (1994) $53-82$.

[18] R. C. Kloosterziel, G. F. Carnevale, On the evolution and saturation of instabilities of two-dimensional isolated circular vortices, J. Fluid. Mech. 388 (1999) 217-257.

[19] F. Gallaire, J. M. Chomaz, Three-dimensional instability of isolated vortices, Phys. Fluids 15 (8) (2003) $2113-2126$.

[20] M. V. Melander, J. C. McWilliams, Z. N. J., The axisymmetrization and vorticity-gradient intensification of an isolated two-dimensional vortex through filamentation, J. Fluid. Mech. 178 (1987) 137-159.

[21] M. V. Melander, N. J. Zabusky, J. C. McWilliams, Symmetric vortex merger in two dimensions: causes and conditions, J. Fluid. Mech. 195 (1988) 303-340.

[22] G. F. Carnevale, J. C. McWilliams, Y. Pomeau, J. B. Weiss, W. R. Young, Evolution of vortex statistics in two-dimensional turbulence, Phys. Rev. Letters 66 (1991) 2735 - 2337.

[23] G. F. Carnevale, P. Cavazza, P. Orlandi, R. Purini, An explanation for anomalous vortex merger in rotating-tank experiments, Physics of Fluids A3 (1997) $1411-1415$

[24] L. Rayleigh, On the dynamics of revolving fluids, Proc. R. Soc. London Ser. A. 93 (1916) 148 - 154.

[25] P. Orlandi, Fluid flow phenomena: a numerical toolkit, Kluwer Academic Publishers, Dordrecht, The Netherlands, 2000.

[26] R. C. Kloosterziel, G. F. Carnevale, Vertical scale selection in inertial instability, J. Fluid. Mech. 594 (2008) $249-269$.

[27] R. C. Kloosterziel, P. Orlandi, G. F. Carnevale, Saturation of inertial instability in rotating planar shear flows, J. Fluid. Mech. 583 (2007) 413-422.

[28] G. F. Carnevale, R. C. Kloosterziel, P. Orlandi, Inertial and barotropic instabilities of a free current in 3d rotating flow, J. Fluid Mech. 725 (2013) $117-151$

[29] R. Plougonven, V. Zeitlin, Nonlinear development of inertial instability in a barotropic shear, Phys. Fluids 21 (2009) 106601-1-106601-15.

[30] F. Bouchut, B. Ribstein, V. Zeitlin, Inertial, barotropic, and baroclinic instabilities of the bickley jet in two-layer rotating shallow water model, Phys. Fluids 23 (2011) 126601-1-126601-22.

[31] R. C. Kloosterziel, P. Orlandi, G. F. Carnevale, Saturation of equatorial inertial instability, J. Fluid Mech. 767 (2015) 562 - 594. 